\title{
A PARAFAC-based algorithm for multidimensional parameter estimation in polarimetric bistatic MIMO radar
}

\author{
Hong Jiang ${ }^{*}$, Yu Zhang, Jia Li and Haijing Cui
}

\begin{abstract}
In this article, we investigate the problem of applying the parallel factor quadrilinear decomposition technique to multidimensional target parameter estimation in a polarimetric bistatic multiple-input multiple-output (MIMO) radar system with a uniform rectangular array at the transmitter and a cross-dipole-based uniform rectangular array at the receiver. The signal model is developed, and a novel algorithm is proposed exploiting the quadrilinear alternating least squares to jointly estimate the two-dimensional direction of departure (2D-DOD), two-dimensional direction of arrival (2D-DOA), polarization parameters and Doppler frequency. Multidimensional parameters can be automatically paired by this algorithm to avoid the performance degradation resulting from wrong pairing. The developed algorithm requires neither multidimensional spectral peak searching nor covariance matrix estimation and several eigen-value decompositions that may bring error accumulation. Furthermore, multiple targets having close 2D-DODs and close 2D-DOAs or even the same 2D-DOD or 2D-DOA are distinguishable by means of polarization diversity. The algorithm improves the performance of multi-target identification and three-dimensional localization. Numerical simulations demonstrate the effectiveness of the proposed algorithm.
\end{abstract}

Keywords: Multidimensional parameter estimation; 2D-DOD; 2D-DOA; Polarization diversity; PARAFAC; Quadrilinear decomposition; Bistatic MIMO radar

\section{Introduction}

In recent years, joint parameter estimation in multipleinput multiple-output (MIMO) radar [1-3] has drawn considerable attention for target identification, localization, imaging, etc. Specifically, bistatic MIMO radar with respectively colocated transmitter and receiver array antennas is widely investigated by many researchers for its capability of jointly estimating the direction of departure (DOD) and direction of arrival (DOA) of targets. Many spatial spectrum estimation approaches have been developed for joint DOD and DOA estimation in a bistatic MIMO radar [4-11]. In [4], a two-dimensional (2D) Capon-based method was developed by searching through all the $2 \mathrm{D}$ space to find the DOA and DOD of a target. By exploiting the invariance property of both the transmitter and receiver arrays, some

*Correspondence: jiangh@jlu.edu.cn

College of Communication Engineering, Jilin University, Changchun 130012, China approaches using the estimation of signal parameter via rotational invariance techniques (ESPRIT) [5-7] have been presented for joint DOA and DOD estimation, avoiding the exhaustive peak searching. However, an additional pair matching between the DOAs and DODs is required for ESPRIT-like methods. Besides, polynomial root finding [8] and combined ESPRIT-multiple signal classification (MUSIC) approach [9] were investigated by decomposing the $2 \mathrm{D}$ angle estimation into double one-dimensional (1D) angle estimation, allowing an automatic pairing between the DOAs and DODs. To reduce computation load, a reduced-dimension MUSIC method was proposed in [10]. Considering the characteristics of non-stationary target signals, joint estimation of DOD and DOA information of maneuvering targets was examined in [11] exploiting spatial timefrequency distribution. For moving targets, the literatures in [12-14] addressed to joint DOD, DOA and Doppler frequency estimation.

\section{Springer}

(c) 2013 Jiang et al.: licensee Springer. This is an Open Access article distributed under the terms of the Creative Commons Attribution License (http://creativecommons.org/licenses/by/2.0), which permits unrestricted use, distribution, and reproduction in any medium, provided the original work is properly cited. 
Notably, the polarization state of a target will change upon reflection. Polarization diversity has been proved to be important in target identification especially when multiple targets are so closely spaced that they cannot be distinguished well in spatial domain. In $[15,16]$, joint DOD-DOA-polarization estimation and joint DOD, 2DDOA and polarization estimation exploiting the ESPRIT technique were proposed for bistatic MIMO radar. The simulations have shown that multiple targets having close DODs or DOAs but different polarizations own different array manifolds and are distinguishable based on their polarization diversity.

Recently, parallel factor (PARAFAC) analysis, as an analysis method of high-dimensional data, has become a new technology applied to signal processing and communication field $[17,18]$. The parallel factor model is a generalization of low-rank decomposition to three- or multi-way arrays, which was first introduced as a data analysis tool in psychometrics. Over the past decades, PARAFAC ideas have been applied in multiple-invariance sensor array processing with emphasis on identifiability results [18]. In recent years, PARAFAC has become a new research means in MIMO radar [19-21]. The PARAFAC analysis algorithms $[19,20]$ and adaptive PARAFAC algorithm [21] have been developed for the estimation of DOAs and DODs of multiple targets.

Despite the fact that several PARAFAC-based methods have been proposed for DOA and DOD estimation in bistatic MIMO radars, trilinear decomposition algorithms [17-21] with trilinear alternating least square (TALS) are commonly used to fit PARAFAC model. In this article, we investigate joint estimation of DOD, DOA, Doppler frequency and polarization. The polarization can be considered as the fourth dimension and hence a four-way description happens to fit better as long as the targets have diverse polarizations. Thus, PARAFAC quadrilinear decomposition [22,23] with quadrilinear alternating least square (QALS) is applied to multidimensional parameter estimation in polarimetric bistatic MIMO radar.

With the restriction that targets can only be located on a $2 \mathrm{D}$ plane in previous algorithms based on 1DDOD and 1D-DOA estimation using uniform linear arrays, the estimation of 2D-DOD and 2D-DOA is investigated in this article exploiting a uniform rectangular array at the transmitter and a cross-dipole-based uniform rectangular array at the receiver. The signal model for polarimetric bistatic MIMO radar is developed, and a novel PARAFAC QALS-based algorithm is presented for joint estimation of seven target parameters, including the 2D-DOA, 2D-DOD, two polarization parameters and Doppler frequency. The proposed algorithm avoids multidimensional spectral peak searching, covariance matrix estimation and several eigen-decompositions that may bring error accumulation, which can enhances the accuracy of estimation. Multidimensional parameter pairing is obtained automatically by this algorithm, which can avoid the performance degradation resulting from wrong pairing. Furthermore, polarization diversity of multiple target characteristics is exploited to distinguish multiple targets having close 2D-DODs and 2D-DOAs or even the same 2D-DOD or 2D-DOA, which can improve the resolution of multi-target identification and 3D localization. The merits of the algorithm are investigated via numerical simulations.

This paper is organized as follows: in the next section, the signal model for polarimetric bistatic MIMO radar is developed. A novel PARAFAC quadrilinear decomposition-based algorithm for 2D-DOA, 2D-DOD, polarizations and Doppler frequency estimation is presented in section 3 . In section 4 , the results of simulation are given to verify the performance of the proposed method. Finally, a conclusion is drawn in section 5 .

\section{Signal model for polarimetric MIMO radar 2.1 Received signal}

As illustrated in Figure 1, consider a polarimetric bistatic MIMO radar equipped with a uniform rectangular transmit array having $M=M_{1} M_{2}$ sensors and a uniform rectangular receive array having $N=N_{1} N_{2}$ crossed dipole sensors, where $M_{1}, M_{2}$ and $N_{1}, N_{2}$ are the numbers of the transmit and receive sensors in the $x$-axis and $y$-axis, respectively. At the receiver array, the crossed dipoles are used to measure the polarization states of a transverse electromagnetic (TEM) wave and exploit polarization diversity. The antennas are of ideal, identical isotropic sensors. The inter-sensor spacings at the transmit and receive arrays are $d_{t}$ and $d_{r}$, respectively, their lengths being no more than half wavelength $(\lambda / 2)$. At the transmit site, $M$ different coded signals with identical bandwidth and center frequency are emitted simultaneously, which are temporally orthogonal. Denote $\mathbf{s}_{m}=$ $\left[s_{m}(1), \cdots, s_{m}(K)\right]$ the $m$ th transmit waveform vector having a code length of $K$ and a unit norm, $m=1, \cdots, M$. There are $P$ targets located in the same range-bin of the receiver site. They are assumed to be point sources. For the $p$ th target, $p=1, \cdots, P$, we denote $\theta_{t p}$ and $\phi_{t p}$ its transmit elevation and azimuth angles (namely, 2D-DOD), $\theta_{r p}$ and $\phi_{r p}$ its receive elevation and azimuth angles (namely, 2D-DOA), $\gamma_{p}$ and $\eta_{p}$ its polarization angle and polarization phase difference, and $f_{d p}$ its Doppler frequency.

Denote $\mathbf{X}_{m, n, p}^{(l)}$ the reflected signal of the $p$ th target from the $m$ th transmit sensor to the $n$th receive sensor in the $l$ th snapshot, $l=1, \cdots, L, m=1, \cdots, M, n=1, \cdots, N$, $p=1, \cdots, P$, and $\mathbf{X}_{1,1, p}^{(l)}$ the reflected signal of the $p$ th target from the reference transmit sensor to the reference 


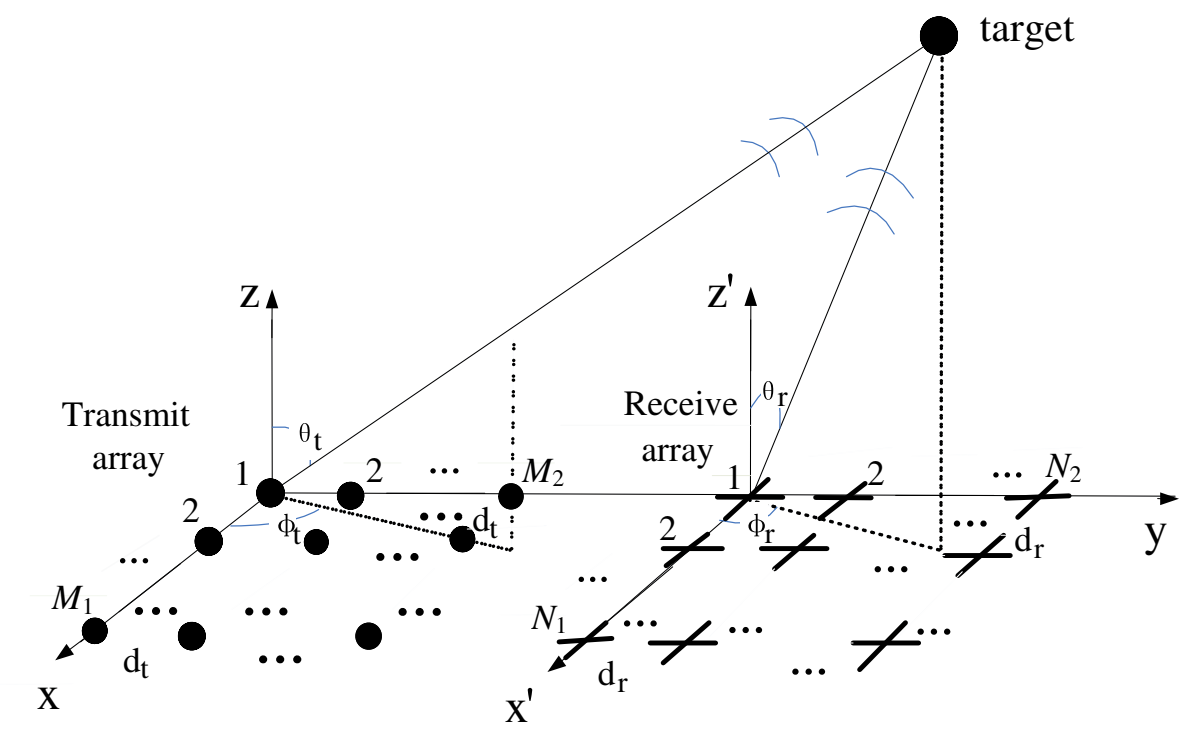

Figure 1 A polarimetric bistatic MIMO radar. With a uniform rectangular array at the transmitter and a cross-dipole uniform rectangular array at the receiver.

receive sensor. The incoming signals of fully polarized electric field TEM wave impinge on the reference receive sensor with crossed dipole antenna. For the $p$ th target, we assume that it arrives at the receive array with the elevation/azimuth angles $\theta_{r p}$ and $\phi_{r p}$. Thus, the observed signal $\mathbf{X}_{1,1, p}^{(l)}$ consists of two polarization components in the $x$ direction and $y$ direction [24], the composite of which can be written as

$$
\begin{aligned}
\mathbf{X}_{1,1, p}^{(l)}= & {\left[\begin{array}{cc}
\cos \theta_{r p} \cos \phi_{r p} & -\sin \phi_{r p} \\
\cos \theta_{r p} \sin \phi_{r p} & \cos \phi_{r p}
\end{array}\right]\left[\begin{array}{c}
\sin \gamma_{p} e^{j \eta_{p}} \\
\cos \gamma_{p}
\end{array}\right] \beta_{p} e^{j 2 \pi f_{d p} t_{l}} } \\
& \times \mathbf{s}_{1}+\mathbf{Z}_{1,1, p}^{(l)}
\end{aligned}
$$

where $\beta_{p}$ denotes the complex amplitude proportional to the radar cross section of the $p$ th target. The target amplitude remains constant in the $L$ snapshots. $t_{l}=$ $l T_{S}$ is the slow time with $T_{s}$ being the duration of a snapshot. $\mathbf{Z}_{1,1, p}^{(l)}$ is the noise measured at the reference sensor. Since the observed signal $\mathbf{X}_{m, n, p}^{(l)}$ coming from the $m$ th transmit sensor to the $n$th receive sensor has the phase shifts relative to $\mathbf{X}_{1,1, p}^{(l)}$, it can be described as

$$
\begin{aligned}
\mathbf{X}_{m, n, p}^{(l)}= & {\left[\begin{array}{cc}
\cos \theta_{r p} \cos \phi_{r p} & -\sin \phi_{r p} \\
\cos \theta_{r p} \sin \phi_{r p} & \cos \phi_{r p}
\end{array}\right]\left[\begin{array}{c}
\sin \gamma_{p} e^{j \eta_{p}} \\
\cos \gamma_{p}
\end{array}\right] } \\
& \times \xi_{r p}^{n_{1}-1} \zeta_{r p}^{n_{2}-1} \xi_{t p}^{m_{1}-1} \zeta_{t p}^{m_{2}-1} \beta_{p} e^{j 2 \pi f_{d p} t_{l}} \mathbf{s}_{m}+\mathbf{Z}_{m, n, p}^{(l)}
\end{aligned}
$$

$m=1, \cdots, M, \quad n=1, \cdots, N, \quad p=1, \cdots, P, \quad m_{i}=$ $1, \cdots, M_{i}, n_{j}=1, \cdots, N_{j}, i, j=1,2$, and $\xi_{t p} \triangleq e^{-j 2 \pi\left(d_{t} / \lambda\right) \sin \theta_{t p} \cos \phi_{t p}}, \quad \zeta_{t p} \triangleq e^{-j 2 \pi\left(d_{t} / \lambda\right) \sin \theta_{t p} \sin \phi_{t p}}$

$\xi_{r p} \triangleq e^{-j 2 \pi\left(d_{r} / \lambda\right) \sin \theta_{r p} \cos \phi_{r p}}, \quad \zeta_{r p} \triangleq e^{-j 2 \pi\left(d_{r} / \lambda\right) \sin \theta_{r p} \sin \phi_{r p}}$

$\mathbf{Z}_{m, n, p}^{(l)}$ is the observed noise. Collect the data of $\mathbf{X}_{m, n, p}^{(l)}$ for $m=1, \cdots, M, n=1, \cdots, N, p=1, \cdots, P$, forming a $2 N \times K$ complex matrix

$$
\begin{aligned}
\mathbf{X}^{(l)}= & \sum_{p=1}^{P} \mathbf{a}_{r}\left(\theta_{r p}, \phi_{r p}\right) \otimes \mathbf{g}\left(\theta_{r p}, \phi_{r p}, \gamma_{p}, \eta_{p}\right) \beta_{p} e^{j 2 \pi f_{d p} t_{l}} \\
& \times \mathbf{a}_{t}^{T}\left(\theta_{t p}, \phi_{t p}\right)\left[\begin{array}{l}
\mathbf{s}_{1} \\
\vdots \\
\mathbf{s}_{M}
\end{array}\right]+\mathbf{Z}^{(l)},
\end{aligned}
$$

where $\otimes$ denotes the Kronecker product, and

$$
\begin{aligned}
\mathbf{g}\left(\theta_{r p}, \phi_{r p}, \gamma_{p}, \eta_{p}\right) \triangleq & {\left[\begin{array}{cc}
\cos \theta_{r p} \cos \phi_{r p} & -\sin \phi_{r p} \\
\cos \theta_{r p} \sin \phi_{r p} & \cos \phi_{r p}
\end{array}\right] } \\
& \times\left[\begin{array}{c}
\sin \gamma_{p} e^{j \eta_{p}} \\
\cos \gamma_{p}
\end{array}\right]
\end{aligned}
$$

is a $2 \times 1$ polarization vector for a crossed dipole antenna array, $0 \leq \gamma_{p} \leq \pi / 2,-\pi \leq \eta_{p}<\pi$. The $p$ th steering vectors with respect to the transmit and receive uniform rectangular arrays are written as $\mathbf{a}_{t}\left(\theta_{t p}, \phi_{t p}\right) \in \mathbb{C}^{M \times 1}$ and $\mathbf{a}_{r}\left(\theta_{r p}, \phi_{r p}\right) \in \mathbb{C}^{N \times 1}$, respectively, with

$\mathbf{a}_{t}\left(\theta_{t p}, \phi_{t p}\right)=\mathbf{a}_{t_{1} p} \otimes \mathbf{a}_{t_{2} p}$,

$\mathbf{a}_{r}\left(\theta_{r p}, \phi_{r p}\right)=\mathbf{a}_{r_{1} p} \otimes \mathbf{a}_{r_{2} p}$, 
where

$$
\begin{aligned}
\mathbf{a}_{t_{1} p} & =\left[1, \xi_{t p}, \cdots, \xi_{t p}^{M_{1}-1}\right]^{T}, \\
\mathbf{a}_{t_{2} p} & =\left[1, \zeta_{t p}, \cdots, \zeta_{t p}^{M_{2}-1}\right]^{T} \\
\mathbf{a}_{r_{1} p} & =\left[1, \xi_{r p}, \cdots, \xi_{r p}^{N_{1}-1}\right]^{T}, \\
\mathbf{a}_{r_{2} p} & =\left[1, \zeta_{r p}, \cdots, \zeta_{r p}^{N_{2}-1}\right]^{T} .
\end{aligned}
$$

The observed matrix in (4) can be rewritten as

$$
\mathbf{X}^{(l)}=\mathbf{A}_{r}\left(\theta_{r}, \phi_{r}\right) \diamond \mathbf{G}\left(\theta_{r}, \phi_{r}, \gamma, \eta\right) \operatorname{diag}\left(\mathbf{b}^{(l)}\right) \mathbf{A}_{t}^{T}\left(\theta_{t}, \phi_{t}\right) \mathbf{S}+\mathbf{Z}^{(l)},
$$

where $\diamond$ represents the Khatri-Rao product (columnwise Kronecker product). $\mathbf{A}_{t}\left(\theta_{t}, \phi_{t}\right), \mathbf{A}_{r}\left(\theta_{r}, \phi_{r}\right)$ and $\mathbf{G}\left(\theta_{r}, \phi_{r}, \gamma, \eta\right)$ denote the transmit steering matrix, receive steering matrix and polarization matrix, respectively. $\mathbf{A}_{t}\left(\theta_{t}, \phi_{t}\right)=\left[\mathbf{a}_{t}\left(\theta_{t 1}, \phi_{t 1}\right), \cdots, \mathbf{a}_{t}\left(\theta_{t P}, \phi_{t P}\right)\right]$, $\mathbf{A}_{r}\left(\theta_{r}, \phi_{r}\right)=\left[\mathbf{a}_{r}\left(\theta_{r 1}, \phi_{r 1}\right), \cdots, \mathbf{a}_{r}\left(\theta_{r P}, \phi_{r P}\right)\right]$, and $\mathbf{G}\left(\theta_{r}\right.$, $\left.\phi_{r}, \gamma, \eta\right)=\left[\mathbf{g}\left(\theta_{r 1}, \phi_{r 1}, \gamma_{1}, \eta_{1}\right), \cdots, \mathbf{g}\left(\theta_{r P}, \phi_{r P}, \gamma_{P}, \eta_{P}\right)\right]$. The matrix $\mathbf{S} \in \mathbb{C}^{M \times K}$ contains $M$ transmit waveforms, $\mathbf{S}=\left[\mathbf{s}_{1}{ }^{T}, \ldots, \mathbf{s}_{M}\right]^{T}$, and $\mathbf{S S}^{H}=\mathbf{I}_{M}$ due to waveform orthogonality and normalization. $\mathbf{Z}^{(l)} \in \mathbb{C}^{2 N \times K}$ denotes the noise matrix, and $\mathbf{b}^{(l)}$ is the target vector, $\mathbf{b}^{(l)}=\left[\beta_{1} e^{j 2 \pi f_{d 1} t_{l}}, \cdots, \beta_{P} e^{j 2 \pi f_{d P} t_{l}}\right]^{T}$.

\subsection{Pulse compression and vectorization}

The received signals are pulse-compressed using $M$ transmit waveforms, forming the output matrix $\widetilde{\mathbf{X}}^{(l)}=\mathbf{X}^{(l)} \mathbf{S}^{H}$, which can be expressed by

$$
\begin{aligned}
\widetilde{\mathbf{X}}^{(l)}= & \mathbf{A}_{r}\left(\theta_{r}, \phi_{r}\right) \diamond \mathbf{G}\left(\theta_{r}, \phi_{r}, \gamma, \eta\right) \operatorname{diag}\left(\mathbf{b}^{(l)}\right) \mathbf{A}_{t}^{T}\left(\theta_{t}, \phi_{t}\right) \\
& +\mathbf{Z}^{(l)} \mathbf{S}^{H},
\end{aligned}
$$

where $\mathbf{Z}^{(l)} \mathbf{S}^{H}$ denotes the noise matrix after pulse compression. Vectorizing $\widetilde{\mathbf{X}}^{(l)}$ in (9) yields the following $2 M N$ column vector:

$$
\mathbf{y}^{(l)}=\operatorname{vec}\left(\tilde{\mathbf{X}}^{(l)}\right)=\mathbf{A}\left(\theta_{t}, \phi_{t}, \theta_{r}, \phi_{r}, \gamma, \eta\right) \mathbf{b}^{(l)}+\mathbf{n}^{(l)},
$$

where $\mathbf{A}\left(\theta_{t}, \phi_{t}, \theta_{r}, \phi_{r}, \gamma, \eta\right)$ denotes the total steering matrix with

$\mathbf{A}\left(\theta_{t}, \phi_{t}, \theta_{r}, \phi_{r}, \gamma, \eta\right)=\mathbf{A}_{t}\left(\theta_{t}, \phi_{t}\right) \diamond \mathbf{A}_{r}\left(\theta_{r}, \phi_{r}\right) \diamond \mathbf{G}\left(\theta_{r}, \phi_{r}, \gamma, \eta\right)$

In (10) and (11), the transformation of vec $(\mathbf{A B C})=$ $\left(\mathbf{C}^{T} \otimes \mathbf{A}\right) \operatorname{vec}(\mathbf{B})$ has been used. Collecting all the observed data of $\mathbf{y}^{(l)}$ over $L$ time slots yields the following $2 M N \times L$ complex matrix

$$
\mathbf{Y}=\mathbf{A}\left(\theta_{t}, \phi_{t}, \theta_{r}, \phi_{r}, \gamma, \eta\right) \mathbf{B}^{T}+\mathbf{N}
$$

with $\mathbf{Y}=\left[\mathbf{y}^{(1)}, \cdots, \mathbf{y}^{(L)}\right]$ and $\mathbf{N}=\left[\mathbf{n}^{(1)}, \cdots, \mathbf{n}^{(L)}\right]$ representing the observed matrix and noise matrix. $\mathbf{B}=$ $\left[\mathbf{b}^{(1)}, \cdots, \mathbf{b}^{(L)}\right]^{T}$ denotes the target matrix, which contains the estimated parameter of Doppler frequency $f_{d}$.

Based on the signal model of polarimetric MIMO radar developed in (12), the problem of interest is to jointly estimate the multidimensional parameters $\theta_{t}, \phi_{t}, \theta_{r}, \phi_{r}, \gamma, \eta$ and $f_{d}$.

\section{PARAFAC quadrilinear decomposition-based algorithm for multidimensional parameter estimation}

In this section, we present the multidimensional parameter estimation algorithm in polarimetric MIMO radar using PARAFAC quadrilinear decomposition.

\subsection{PARAFAC quadrilinear decomposition model and uniqueness theorem}

The definition of PARAFAC quadrilinear decomposition model can be derivatively described from the trilinear decomposition model [19-21].

Definition 1. (Quadrilinear decomposition in tensor format) A quadrilinear decomposition of a four-order tensor $\underline{\mathbf{X}} \in \mathbb{C}^{I \times J \times K \times L}$ is a decomposition of the type $\underline{\mathbf{X}}=$ $\sum_{m=1}^{M} \mathbf{a}_{m} \circ \mathbf{b}_{m} \circ \mathbf{c}_{m} \circ \mathbf{d}_{m}$, where $\mathbf{a}_{m}, \mathbf{b}_{m}, \mathbf{c}_{m}, \mathbf{d}_{m}$ are the $m$ th columns of the matrices $\mathbf{A} \in \mathbb{C}^{I \times M}, \mathbf{B} \in \mathbb{C}^{J \times M}, \mathbf{C} \in$ $\mathbb{C}^{K \times M}$ and $\mathbf{D} \in \mathbb{C}^{L \times M}$, respectively, and $\circ$ is the outer product, i.e., $\left(\mathbf{a}_{m} \circ \mathbf{b}_{m} \circ \mathbf{c}_{m} \circ \mathbf{d}_{m}\right)_{i j k l}=a_{i m} b_{j m} c_{k m} d_{l m}$, for all values of the indices $i, j, k$ and $l$.

Definition 2. (Quadrilinear decomposition in matrix format) Let $\mathbf{X}^{(1)} \in \mathbb{C}^{I J K \times L}, \mathbf{X}^{(2)} \in \mathbb{C}^{L I J \times K}, \mathbf{X}^{(3)} \in \mathbb{C}^{K L I \times J}$ and $\mathbf{X}^{(4)} \in \mathbb{C}^{J K L \times I}$ be the four matrix representations of a four-way array. The quadrilinear decomposition of $\underline{\mathbf{X}}$ can be written as four equivalent matrices $\mathbf{X}^{(1)}=(\mathbf{A} \diamond \mathbf{B} \diamond$ $\mathbf{C}) \mathbf{D}^{T}, \mathbf{X}^{(2)}=(\mathbf{D} \diamond \mathbf{A} \diamond \mathbf{B}) \mathbf{C}^{T}, \mathbf{X}^{(3)}=(\mathbf{C} \diamond \mathbf{D} \diamond \mathbf{A}) \mathbf{B}^{T}$ and $\mathbf{X}^{(4)}=(\mathbf{B} \diamond \mathbf{C} \diamond \mathbf{D}) \mathbf{A}^{T}$.

Under certain conditions, $\underline{\mathbf{X}}$ can be decomposed uniquely into matrices $\mathbf{A}, \mathbf{B}, \mathbf{C}$ and $\mathbf{D}$. These conditions are based on the notion of Kruskal-rank $[17,18]$.

Definition 3. (Kruskal-rank or k-rank) [18] Consider a matrix $\mathbf{A} \in \mathbb{C}^{I \times M}$. If $\operatorname{rank}(\mathbf{A})=r$, then $\mathbf{A}$ contains a collection of $r$ linearly independent columns. Moreover, if every $k<M$ columns of $\mathbf{A}$ are linearly independent, but this does not hold for every $k+1$ columns, then $\mathbf{A}$ has $k$-rank $k_{\mathbf{A}}=k$. Note that $k_{\mathbf{A}}<\operatorname{rank}(\mathbf{A}), \forall \mathbf{A}$.

Theorem 1. (Uniqueness of quadrilinear decomposition) [25] Consider that a matrix representation of $\underline{\mathbf{X}}$ is $\mathbf{X}=(\mathbf{A} \diamond \mathbf{B} \diamond \mathbf{C}) \mathbf{D}^{T}$, where $\mathbf{A} \in \mathbb{C}^{I \times M}, \mathbf{B} \in \mathbb{C}^{J \times M}, \overline{\mathbf{C}} \in$ 
$\mathbb{C}^{K \times M}$ and $\mathbf{D} \in \mathbb{C}^{L \times M}$ are the four mode matrices of $\underline{\mathbf{X}}$, and $M$ denotes the common dimension. If

$$
k_{\mathbf{A}}+k_{\mathbf{B}}+k_{\mathbf{C}}+k_{\mathbf{D}} \geq 2 M+3,
$$

then $\mathbf{A}, \mathbf{B}, \mathbf{C}$ and $\mathbf{D}$ are unique up to permutation and scaling of columns, meaning that any other quadruple $\widetilde{\mathbf{A}}, \widetilde{\mathbf{B}}$, $\widetilde{\mathbf{C}}$ and $\widetilde{\mathbf{D}}$ that gives rise to $\mathbf{X}$ is related to $\mathbf{A}, \mathbf{B}, \mathbf{C}, \mathbf{D}$ via $\widetilde{\mathbf{A}}=\mathbf{A} \Pi \boldsymbol{\Lambda}_{1}, \widetilde{\mathbf{B}}=\mathbf{B} \Pi \boldsymbol{\Lambda}_{2}, \widetilde{\mathbf{C}}=\mathbf{C} \Pi \boldsymbol{\Lambda}_{3}, \widetilde{\mathbf{D}}=\mathbf{C} \Pi \boldsymbol{\Lambda}_{4}$ where $\boldsymbol{\Pi}$ is a permutation matrix, $\boldsymbol{\Lambda}_{1}, \boldsymbol{\Lambda}_{2}, \boldsymbol{\Lambda}_{3}$ and $\boldsymbol{\Lambda}_{4}$ are diagonal scaling matrices satisfying $\Lambda_{1} \Lambda_{2} \Lambda_{3} \Lambda_{4}=\mathbf{I}_{M}$.

\subsection{Parameter matrix estimation using QALS}

In this subsection, QALS algorithm is applied to uniquely identify the four parameter matrices $\mathbf{A}_{t}\left(\theta_{t}, \phi_{t}\right), \mathbf{A}_{r}\left(\theta_{r}, \phi_{r}\right)$, $\mathbf{G}\left(\theta_{r}, \phi_{r}, \gamma, \eta\right)$ and $\mathbf{B}\left(f_{d}\right)$ for multidimensional parameter estimation. We write the observed matrix $\mathbf{Y}$ in (12) as follows:

$$
\mathbf{Y}=\mathbf{A}_{t}\left(\theta_{t}, \phi_{t}\right) \diamond \mathbf{A}_{r}\left(\theta_{r}, \phi_{r}\right) \diamond \mathbf{G}\left(\theta_{r}, \phi_{r}, \gamma, \eta\right) \mathbf{B}^{T}\left(f_{d}\right)+\mathbf{N}
$$

with $\mathbf{A}_{t}\left(\theta_{t}, \phi_{t}\right) \in \mathbb{C}^{M \times P}, \mathbf{A}_{r}\left(\theta_{r}, \phi_{r}\right) \in \mathbb{C}^{N \times P}, \mathbf{G}\left(\theta_{r}, \phi_{r}, \gamma, \eta\right)$ $\in \mathbb{C}^{2 \times P}, \mathbf{B}\left(f_{d}\right) \in \mathbb{C}^{L \times P}$.

Based on definition 2, it is clear that (14) corresponds to one of the matrix representations of quadrilinear decomposition of a four-way array $\underline{\mathbf{Y}} \in \mathbb{C}^{M \times N \times 2 \times L}$, with $P$ denoting the common dimension. For clear analysis, we express the $2 M N \times L$ matrix $\mathbf{Y}$ as $\mathbf{Y}^{(1)}$. Thus, quadrilinear decomposition can obtain an equivalent $L M N \times 2$ matrix representation as the following form:

$$
\mathbf{Y}^{(2)}=\mathbf{B}\left(f_{d}\right) \diamond \mathbf{A}_{t}\left(\theta_{t}, \phi_{t}\right) \diamond \mathbf{A}_{r}\left(\theta_{r}, \phi_{r}\right) \mathbf{G}^{T}\left(\theta_{r}, \phi_{r}, \gamma, \eta\right)+\mathbf{N}_{2}
$$

an equivalent $2 L M \times N$ matrix representation

$$
\mathbf{Y}^{(3)}=\mathbf{G}\left(\theta_{r}, \phi_{r}, \gamma, \eta\right) \diamond \mathbf{B}\left(f_{d}\right) \diamond \mathbf{A}_{t}\left(\theta_{t}, \phi_{t}\right) \mathbf{A}_{r}{ }^{T}\left(\theta_{r}, \phi_{r}\right)+\mathbf{N}_{3}
$$

and an equivalent $2 L N \times M$ matrix representation

$$
\mathbf{Y}^{(4)}=\mathbf{A}_{r}\left(\theta_{r}, \phi_{r}\right) \diamond \mathbf{G}\left(\theta_{r}, \phi_{r}, \gamma, \eta\right) \diamond \mathbf{B}\left(f_{d}\right) \mathbf{A}_{t}^{T}\left(\theta_{t}, \phi_{t}\right)+\mathbf{N}_{4},
$$

where $\mathbf{Y}^{(1)}, \mathbf{Y}^{(2)}, \mathbf{Y}^{(3)}$ and $\mathbf{Y}^{(4)}$ denote the slice sets of the four-way array $\underline{\mathbf{Y}}$ along the snapshot, polarization, reception and transmission ways, respectively.

From definition 3 and theorem $I$ in section 3.1, it is obvious that the k-ranks of $\mathbf{A}_{t}, \mathbf{A}_{r}, \mathbf{G}$ and $\mathbf{B}$ are $k_{\mathbf{A}_{t}}=k_{\mathbf{A}_{r}}=P$, $k_{\mathbf{G}}=\min (2, P), k_{\mathbf{B}}=\min (L, P)$, respectively. If multiple targets and enough snapshots are taken into account, i.e. $L \geq P \geq 2$, then the following inequality is always satisfied:

$$
k_{\mathbf{A}_{t}}+k_{\mathbf{A}_{r}}+k_{\mathbf{G}}+k_{\mathbf{B}}=P+P+2+P \geq 2 P+3,
$$

which means that the four-way array $\underline{\mathbf{Y}}$ satisfies the Kruskal-rank Theorem. Thus, $\mathbf{A}_{t}, \mathbf{A}_{r}, \overline{\mathbf{G}}$ and $\mathbf{B}$ can be recovered uniquely up to permutation and scaling ambiguity.

Based on the four matrix representations $\mathbf{Y}^{(1)}, \mathbf{Y}^{(2)}$, $\mathbf{Y}^{(3)}$ and $\mathbf{Y}^{(4)}$, the parameter matrices $\mathbf{A}_{t}\left(\theta_{t}, \phi_{t}\right), \mathbf{A}_{r}\left(\theta_{r}, \phi_{r}\right)$, $\mathbf{G}\left(\theta_{r}, \phi_{r}, \gamma, \eta\right)$ and $\mathbf{B}\left(f_{d}\right)$ can be estimated using quadrilinear alternating least square (QALS) to fit PARAFAC model. The basic idea behind QALS is to update one parameter matrix using the least squares algorithm, conditioned on previously obtained estimates for the remaining parameter matrices that define the decomposition. This process is repeated until convergence in the least squares fit. The detailed iterative algorithm is as follows:

i) Initialize the estimation $\hat{\mathbf{A}}_{t}\left(\theta_{t}, \phi_{t}\right), \hat{\mathbf{A}}_{r}\left(\theta_{r}, \phi_{r}\right)$, $\hat{\mathbf{G}}\left(\theta_{r}, \phi_{r}, \gamma, \eta\right)$ and $\hat{\mathbf{B}}\left(f_{d}\right)$ with random matrices, denoting them as $\hat{\mathbf{A}}_{t 0}\left(\theta_{t}, \phi_{t}\right), \hat{\mathbf{A}}_{r 0}\left(\theta_{r}, \phi_{r}\right)$, $\hat{\mathbf{G}}_{0}\left(\theta_{r}, \phi_{r}, \gamma, \eta\right)$ and $\hat{\mathbf{B}}_{0}\left(f_{d}\right)$. The iteration number is $k=1,2,3, \cdots$.

ii) Given $\hat{\mathbf{A}}_{t(k-1)}\left(\theta_{t}, \phi_{t}\right), \hat{\mathbf{A}}_{r(k-1)}\left(\theta_{r}, \phi_{r}\right)$ and $\hat{\mathbf{G}}_{(k-1)}\left(\theta_{r}, \phi_{r}, \gamma, \eta\right)$, obtain the least squares solution of the $k$ th iteration of $\hat{\mathbf{B}}_{k}\left(f_{d}\right)$ according to

$$
\begin{aligned}
\hat{\mathbf{B}}_{k}\left(f_{d}\right)= & \underset{\hat{\mathbf{B}}}{\arg \min } \| \mathbf{Y}^{(1)}-\hat{\mathbf{A}}_{t(k-1)}\left(\theta_{t}, \phi_{t}\right) \diamond \hat{\mathbf{A}}_{r(k-1)}\left(\theta_{r}, \phi_{r}\right) \\
& \diamond \hat{\mathbf{G}}_{(k-1)}\left(\theta_{r}, \phi_{r}, \gamma, \eta\right) \hat{\mathbf{B}}_{k}^{T}\left(f_{d}\right) \|_{F},
\end{aligned}
$$

where \|\|$_{F}$ denote the Frobenius norm of its matrix argument.

iii) Update the $k$ th iteration solution $\hat{\mathbf{G}}_{k}\left(\theta_{r}, \phi_{r}, \gamma, \eta\right)$ based on $\hat{\mathbf{B}}_{k}\left(f_{d}\right), \hat{\mathbf{A}}_{t(k-1)}\left(\theta_{t}, \phi_{t}\right)$ and $\hat{\mathbf{A}}_{r(k-1)}\left(\theta_{r}, \phi_{r}\right)$, such that

$$
\begin{aligned}
\hat{\mathbf{G}}_{k}\left(\theta_{r}, \phi_{r}, \gamma, \eta\right)= & \underset{\hat{\mathbf{G}}}{\arg \min } \| \mathbf{Y}^{(2)}-\hat{\mathbf{B}}_{k}\left(f_{d}\right) \diamond \hat{\mathbf{A}}_{t(k-1)}\left(\theta_{t}, \phi_{t}\right) \\
& \diamond \hat{\mathbf{A}}_{r(k-1)}\left(\theta_{r}, \phi_{r}\right) \hat{\mathbf{G}}_{k}^{T}\left(\theta_{r}, \phi_{r}, \gamma, \eta\right) \|_{F}
\end{aligned}
$$

iv) Update the $k$ th iteration solution $\hat{\mathbf{A}}_{r k}\left(\theta_{r}, \phi_{r}\right)$ based on $\hat{\mathbf{G}}_{k}\left(\theta_{r}, \phi_{r}, \gamma, \eta\right), \hat{\mathbf{B}}_{k}\left(f_{d}\right)$ and $\hat{\mathbf{A}}_{t(k-1)}\left(\theta_{t}, \phi_{t}\right)$, such that

$$
\begin{aligned}
\hat{\mathbf{A}}_{r k}\left(\theta_{r}, \phi_{r}\right)= & \underset{\hat{\mathbf{A}}_{r}}{\arg \min } \| \mathbf{Y}^{(3)}-\hat{\mathbf{G}}_{k}\left(\theta_{r}, \phi_{r}, \gamma, \eta\right) \diamond \hat{\mathbf{B}}_{k}\left(f_{d}\right) \\
& \diamond \hat{\mathbf{A}}_{t(k-1)}\left(\theta_{t}, \phi_{t}\right) \hat{\mathbf{A}}_{r k}^{T}\left(\theta_{r}, \phi_{r}\right) \|_{F}
\end{aligned}
$$


v) Substitute the estimated $\hat{\mathbf{A}}_{r k}\left(\theta_{r}, \phi_{r}\right), \hat{\mathbf{G}}_{k}\left(\theta_{r}, \phi_{r}, \gamma, \eta\right)$ and $\hat{\mathbf{B}}_{k}\left(f_{d}\right)$ into

$$
\begin{aligned}
\hat{\mathbf{A}}_{t k}\left(\theta_{t}, \phi_{t}\right)= & \underset{\hat{\mathbf{A}}_{t}}{\arg \min } \| \mathbf{Y}^{(4)}-\hat{\mathbf{A}}_{r k}\left(\theta_{r}, \phi_{r}\right) \diamond \hat{\mathbf{G}}_{k}\left(\theta_{r}, \phi_{r}, \gamma, \eta\right) \\
& \diamond \hat{\mathbf{B}}_{k}\left(f_{d}\right) \hat{\mathbf{A}}_{t k}^{T}\left(\theta_{t}, \phi_{t}\right) \|_{F}
\end{aligned}
$$

acquiring the least squares solution of the $k$ th iteration $\hat{\mathbf{A}}_{t k}\left(\theta_{t}, \phi_{t}\right)$.

vi) Compute the error $\delta_{k}=\| \mathbf{Y}^{(1)}-\hat{\mathbf{A}}_{t k}\left(\theta_{t}, \phi_{t}\right) \diamond \hat{\mathbf{A}}_{r k}\left(\theta_{r}\right.$, $\left.\phi_{r}\right) \diamond \hat{\mathbf{G}}_{k}\left(\theta_{r}, \phi_{r}, \gamma, \eta\right) \hat{\mathbf{B}}_{k}^{T}\left(f_{d}\right) \|_{F}$ at the kth iteration. The algorithm has converged when $\left|\delta_{k}-\delta_{k-1}\right| \leq \varepsilon$, where $\varepsilon$ is an error threshold.

\subsection{Multidimensional parameter estimation}

Upon obtaining the estimates of $\hat{\mathbf{A}}_{t}\left(\theta_{t}, \phi_{t}\right), \hat{\mathbf{A}}_{r}\left(\theta_{r}, \phi_{r}\right)$, $\hat{\mathbf{G}}\left(\theta_{r}, \phi_{r}, \gamma, \eta\right)$ and $\hat{\mathbf{B}}\left(f_{d}\right)$, the following problem is to estimate multidimensional parameters of $P$ targets from these parameter matrices. The algorithm is based on available Vandermonde structure [26] and Kronecker product structure in the matrix blocks, also uses the polarizationsensitive array processing technology [27].

\subsubsection{D-DOD estimation}

The obtained matrix $\hat{\mathbf{A}}_{t}\left(\theta_{t}, \phi_{t}\right)$ contains the estimated 2DDOD. According to (6), the $p$ th column of $\mathbf{A}_{t}\left(\theta_{t}, \phi_{t}\right)$ for $p=1, \cdots, P$ is the Kronecker product of $\mathbf{a}_{t_{1} p}$ and $\mathbf{a}_{t_{2} p}$. Thus, $\mathbf{A}_{t}\left(\theta_{t}, \phi_{t}\right)$ can be further expressed as

$$
\mathbf{A}_{t}\left(\theta_{t}, \phi_{t}\right)=\left[\begin{array}{cccc}
\mathbf{a}_{t_{2} 1} & \cdots & \cdots & \mathbf{a}_{t_{2} P} \\
\xi_{t 1} \mathbf{a}_{t_{2} 1} & \cdots & \cdots & \xi_{t P} \mathbf{a}_{t_{2} P} \\
\vdots & \vdots & \vdots & \vdots \\
\xi_{t 1}^{M_{1}-1} \mathbf{a}_{t_{2} 1} & \cdots & \cdots & \xi_{t P}^{M_{1}-1} \mathbf{a}_{t_{2} P}
\end{array}\right]
$$

with $\mathbf{a}_{t_{2} p}=\left[1, \zeta_{t p}, \zeta_{t p}^{2}, \cdots, \zeta_{t p}^{M_{2}-1}\right]^{T}$. Equation 23 reveals that $\mathbf{A}_{t}\left(\theta_{t}, \phi_{t}\right)$ is composed of $M_{1}$ blocks according to rows, each block being a $M_{2} \times P$ matrix with Vandermonde structure. $\left[\xi_{t 1}, \cdots, \xi_{t P}\right]$ is the ratio between the corresponding elements of the $\left(m_{1}+1\right)$ th and $m_{1}$ th blocks of $\mathbf{A}_{t}\left(\theta_{t}, \phi_{t}\right)$ for $m_{1}=1, \cdots, M_{1}-1$, and $\left[\zeta_{t 1}, \cdots, \zeta_{t P}\right]$ is the ratio between the corresponding elements of the $\left(m_{2}+1\right)$ th and $m_{2}$ th rows in each block of $\mathbf{A}_{t}\left(\theta_{t}, \phi_{t}\right)$, $m_{2}=1, \cdots, M_{2}-1$. Therefore, calculating the average of $M_{1}\left(M_{2}-1\right)$ ratios respectively yields

$$
\hat{\xi}_{t p}=\frac{1}{M_{1}\left(M_{2}-1\right)} \sum_{m=1}^{M_{1}\left(M_{2}-1\right)} \frac{\left[\hat{\mathbf{A}}_{t}\left(\theta_{t}, \phi_{t}\right)\right]_{m+M_{2}, p}}{\left[\hat{\mathbf{A}}_{t}\left(\theta_{t}, \phi_{t}\right)\right]_{m, p}}
$$

$$
\hat{\zeta}_{t p}=\frac{1}{M_{1}\left(M_{2}-1\right)} \sum_{m_{1}=1}^{M_{1}} \sum_{m_{2}=1}^{M_{2}-1} \frac{\left[\hat{\mathbf{A}}_{t}\left(\theta_{t}, \phi_{t}\right)\right]_{\left(m_{1}-1\right) M_{1}+m_{2}+1, p}}{\left[\hat{\mathbf{A}}_{t}\left(\theta_{t}, \phi_{t}\right)\right]_{\left(m_{1}-1\right) M_{1}+m_{2}, p}},
$$

where $[\mathbf{A}]_{i, j}$ denotes the $(i, j)$ element of the matrix A, and $\hat{\xi}_{t p}$ and $\hat{\zeta}_{t p}$ are the estimates of $\xi_{t p}$ and $\zeta_{t p}$, respectively. From (24) and (25), we have $\sqrt{\operatorname{angle}^{2}\left(\hat{\xi}_{t p}\right)+\operatorname{angle}^{2}\left(\hat{\zeta}_{t p}\right)}=2 \pi\left(d_{t} / \lambda\right) \sin \left(\hat{\theta}_{t p}\right)$, and $\frac{\text { angle }\left(\hat{\zeta}_{t p}\right)}{\operatorname{angle}\left(\hat{\xi}_{t p}\right)}=\tan \left(\hat{\phi}_{t p}\right)$, where angle $(a)$ denotes the phase of a complex number $a$ and $\hat{\theta}_{t p}$ and $\hat{\phi}_{t p}$ are the estimates of $\theta_{t p}$ and $\phi_{t p}$, respectively. Thus, the estimates of 2D-DOD can be calculated if given $\hat{\xi}_{t p}$ and $\hat{\xi}_{t p}$. For $0 \leq \hat{\theta}_{t p}<\pi / 2$ and $-\pi / 2 \leq \hat{\phi}_{t p}<\pi / 2$, we have

$$
\begin{aligned}
& \hat{\theta}_{t p}=\sin ^{-1}\left[\frac{\lambda}{2 \pi d_{t}} \sqrt{\operatorname{angle}^{2}\left(\hat{\xi}_{t p}\right)+\operatorname{angle}^{2}\left(\hat{\zeta}_{t p}\right)}\right] \\
& \hat{\phi}_{t p}=\tan ^{-1}\left[\frac{\operatorname{angle}\left(\hat{\zeta}_{t p}\right)}{\operatorname{angle}\left(\hat{\xi}_{t p}\right)}\right] .
\end{aligned}
$$

\subsubsection{D-DOA estimation}

Similarly, the parameters of 2D-DOA can be attained from the matrix $\hat{\mathbf{A}}_{r}\left(\theta_{r}, \phi_{r}\right)$. The estimates of $\theta_{r p}$ and $\phi_{r p}$ are respectively calculated by

$$
\begin{aligned}
& \hat{\theta}_{r p}=\sin ^{-1}\left[\frac{\lambda}{2 \pi d_{r}} \sqrt{\operatorname{angle}^{2}\left(\hat{\xi}_{r p}\right)+\operatorname{angle}^{2}\left(\hat{\zeta}_{r p}\right)}\right] \\
& \hat{\phi}_{r p}=\tan ^{-1}\left[\frac{\operatorname{angle}\left(\hat{\zeta}_{r p}\right)}{\operatorname{angle}\left(\hat{\xi}_{r p}\right)}\right]
\end{aligned}
$$

for $0 \leq \hat{\theta}_{r p}<\pi / 2$ and $-\pi / 2 \leq \hat{\phi}_{r p}<\pi / 2$, where $\hat{\xi}_{r p}$ and $\hat{\zeta}_{r p}$ are the estimates of $\xi_{r p}$ and $\zeta_{r p}$, respectively, which can be obtained by

$$
\begin{gathered}
\hat{\xi}_{r p}=\frac{1}{N_{1}\left(N_{2}-1\right)} \sum_{n=1}^{N_{1}\left(N_{2}-1\right)} \frac{\left[\hat{\mathbf{A}}_{r}\left(\theta_{r}, \phi_{r}\right)\right]_{n+N_{2}, p}}{\left[\hat{\mathbf{A}}_{r}\left(\theta_{r}, \phi_{r}\right)\right]_{n, p}} \\
\hat{\zeta}_{r p}=\frac{1}{N_{1}\left(N_{2}-1\right)} \sum_{n_{1}=1}^{N_{1}} \sum_{n_{2}=1}^{N_{2}-1} \frac{\left[\hat{\mathbf{A}}_{r}\left(\theta_{r}, \phi_{r}\right)\right]_{\left(n_{1}-1\right) N_{1}+n_{2}+1, p}}{\left[\hat{\mathbf{A}}_{r}\left(\theta_{r}, \phi_{r}\right)\right]_{\left(n_{1}-1\right) N_{1}+n_{2}, p}}
\end{gathered}
$$




\subsubsection{Polarization parameter estimation}

Upon obtaining $\hat{\theta}_{r p}$ and $\hat{\phi}_{r p}$, the estimates of two polarization parameters $\gamma_{p}$ and $\eta_{p}$ can be calculated based on the polarization matrix $\hat{\mathbf{G}}\left(\theta_{r}, \phi_{r}, \gamma, \eta\right)$. From (5), the $p$ th column of $\mathbf{G}\left(\theta_{r}, \phi_{r}, \gamma, \eta\right)$ can be rewritten as

$\mathbf{g}\left(\theta_{r p}, \phi_{r p}, \gamma_{p}, \eta_{p}\right)=\left[\begin{array}{c}\sin \gamma_{p} \cos \theta_{r p} \cos \phi_{r p} e^{j \eta_{p}}-\cos \gamma_{p} \sin \phi_{r p} \\ \sin \gamma_{p} \cos \theta_{r p} \sin \phi_{r p} e^{j \eta_{p}}+\cos \gamma_{p} \cos \phi_{r p}\end{array}\right]$.

Define $\varsigma_{p}$ as the ratio between the second and first elements of the polarization vector $\mathbf{g}\left(\theta_{r p}, \phi_{r p}, \gamma_{p}, \eta_{p}\right)$ in (32)

$$
\varsigma_{p} \triangleq \frac{\sin \gamma_{p} \cos \theta_{r p} \sin \phi_{r p} e^{j \eta_{p}}+\cos \gamma_{p} \cos \phi_{r p}}{\sin \gamma_{p} \cos \theta_{r p} \cos \phi_{r p} e^{j \eta_{p}}-\cos \gamma_{p} \sin \phi_{r p}}
$$

The estimate of $\varsigma_{p}$ can be calculated by

$$
\hat{\zeta}_{p}=\frac{\left[\hat{\mathbf{G}}\left(\theta_{r}, \phi_{r}, \gamma, \eta\right)\right]_{2, p}}{\left[\hat{\mathbf{G}}\left(\theta_{r}, \phi_{r}, \gamma, \eta\right)\right]_{1, p}}
$$

where $\hat{\zeta}_{p}$ is the estimate of $\varsigma_{p}$. Rewriting $\varsigma_{p}$ in (33) yields

$$
\varsigma_{p}=\frac{\tan \gamma_{p} \cos \theta_{r p} \sin \phi_{r p} e^{j \eta_{p}}+\cos \phi_{r p}}{\tan \gamma_{p} \cos \theta_{r p} \cos \phi_{r p} e^{j \eta_{p}}-\sin \phi_{r p}}
$$

Then we have

$$
\tan \gamma_{p} e^{j \eta_{p}}=\frac{\varsigma_{p} \sin \phi_{r p}+\cos \phi_{r p}}{\cos \theta_{r p}\left(\varsigma_{p} \cos \phi_{r p}-\sin \phi_{r p}\right)} .
$$

Since $\tan \gamma_{p} \geq 0$ for $0 \leq \gamma_{p} \leq \pi / 2$, and $\cos \theta_{r p}>0$ for $0 \leq \theta_{r p}<\pi / 2$, the estimates of polarization parameters $\gamma_{p}$ and $\eta_{p}$ can be calculated by

$$
\begin{aligned}
& \hat{\gamma}_{p}=\tan ^{-1}\left(\left|\frac{\hat{\zeta}_{p} \sin \hat{\phi}_{r p}+\cos \hat{\phi}_{r p}}{\cos \hat{\theta}_{r p}\left(\hat{\zeta}_{p} \cos \hat{\phi}_{r p}-\sin \hat{\phi}_{r p}\right)}\right|\right) \\
& \hat{\eta}_{p}=\operatorname{angle}\left(\frac{\hat{\zeta}_{p} \sin \hat{\phi}_{r p}+\cos \hat{\phi}_{r p}}{\hat{\zeta}_{p} \cos \hat{\phi}_{r p}-\sin \hat{\phi}_{r p}}\right) .
\end{aligned}
$$

\subsubsection{Doppler frequency estimation}

The estimate of Doppler frequency is derived from the matrix $\hat{\mathbf{B}}\left(f_{d}\right)$. Define $\omega_{p} \triangleq e^{j 2 \pi f_{d p} T_{s}}$, then

$$
\mathbf{B}\left(f_{d}\right)=\left[\begin{array}{cccc}
\beta_{1} \omega_{1} & \cdots & \cdots & \beta_{P} \omega_{P} \\
\beta_{1} \omega_{1}^{2} & \cdots & \cdots & \beta_{P} \omega_{P}^{2} \\
\vdots & \vdots & \vdots & \vdots \\
\beta_{1} \omega_{1}^{L} & \cdots & \cdots & \beta_{P} \omega_{P}^{L}
\end{array}\right] .
$$

Since $\left[\omega_{1}, \cdots, \omega_{P}\right]$ is the ratio between the $(l+1)$ th and $l$ th rows of the matrix $\mathbf{B}\left(f_{d}\right)$ for $l=1, \cdots, L$, perform the average of ratios yielding

$$
\hat{\omega}_{p}=\frac{1}{L-1} \sum_{l=1}^{L-1} \frac{\left[\hat{\mathbf{B}}\left(f_{d}\right)\right]_{l+1, p}}{\left[\hat{\mathbf{B}}\left(f_{d}\right)\right]_{l, p}}
$$

where $\hat{\omega}_{p}$ is the estimate of $\omega_{p}$. Therefore, the Doppler frequency can be estimated by

$$
\hat{f}_{d p}=\frac{1}{2 \pi T_{s}} \text { angle }\left(\hat{\omega}_{p}\right)
$$

Upon the above analysis, multidimensional parameter $\theta_{t}, \phi_{t}, \theta_{r}, \phi_{r}, \gamma, \eta$ and $f_{d}$ for the $p$ th target can be effectively calculated, $p=1, \cdots, P$.

\subsection{Discussion}

Note that the proposed method can obtain unique parametric identification even when multiple targets have close 2D-DODs or close 2D-DOAs while they are diversely polarized. Specifically, we consider the situation that $P$ targets have the same $2 \mathrm{D}-\mathrm{DOD}$ or the same $2 \mathrm{D}$ DOA, while the other parameters are different. In this case, the inequality in (18) becomes

$$
k_{\mathbf{A}_{t}}+k_{\mathbf{A}_{r}}+k_{\mathbf{G}}+k_{\mathbf{B}}=1+P+2+P=P+1+2+P=2 P+3 .
$$

From the Kruskal-rank Theorem in section 3.1, the sufficient condition is derived herein to guarantee that the quadrilinear decomposition model is unique. Therefore, with polarization diversity, the 2D-DODs and 2D-DOAs of multiple closely spaced targets in MIMO radar can be uniquely identified, and high-resolution estimation can be achieved. It is mentioned that, based on the signal model described in (14), the trilinear decomposition-based algorithm with TALS can also be applied to identify multidimensional parameters using three matrices $\mathbf{A}_{t}, \mathbf{A}_{r} \diamond \mathbf{G}$ and $\mathbf{B}$ as the slice sets of three-way array $\underline{\mathbf{Y}}$. However, for trilinear decomposition, the Kruskal-rank condition can not be satisfied in the situation that $P$ targets have the same 2DDOD, since the inequality $k_{\mathbf{A}_{t}}+k_{\mathbf{A}_{r} \diamond \mathbf{G}}+k_{\mathbf{B}} \geq 2 P+2$ is needed to uniquely identify the three parameter matrices. 
Besides, robust iterative fitting of multilinear approaches such as linear programming (LP) or weighted median filtering iteration [28] can also be applied here to further yield a better solution for robust estimation in nonGaussian noise.

The following assumption is made in the article: (i) The antennas are assumed to be ideal, identical isotropic scatterers. In practical MIMO radar systems, the effect of mutual coupling and array self-calibration methods should be considered. (ii) The target amplitude is assumed to remain constant during snapshot collection (Swerling I model). The condition can be relaxed to Swerling II model [29] when Doppler shift is known. (iii) The number of targets is assumed to be known. In practical situation, the detection of the number of targets, such as the minimum description length (MDL) criterion, should be investigated to obtain the rank of the four-way array $\underline{\mathbf{Y}}$. (iv) The targets are assumed to be point sources. In practical MIMO radar implementation, distributed target models and methods can be further considered.

\section{Simulation}

The simulations are conducted to verify the effectiveness of the proposed method in this section. Consider a polarimetric bistatic MIMO radar system with $M_{1}=M_{2}=3$ and $N_{1}=N_{2}=3$. The inter-sensor spacings are $d_{t}=$ $d_{r}=\lambda / 2$. The duration of a snapshot is $T_{s}=50 \mu \mathrm{s}$, and the length of transmit codes is $K=1024$. The amplitudes of $P$ targets are $\beta_{1}=\beta_{2}=\cdots \beta_{P}=1$. The number of snapshots is $L=100$, and Monte Carlo trials are 100 .

Simulation 1. Consider $P=3$ targets with 2D-DOD, 2D-DOA, polarization parameters and Doppler frequency respectively being $\left(\theta_{t 1}, \theta_{t 2}, \theta_{t 3}\right)=\left(10^{\circ}, 30^{\circ}, 70^{\circ}\right),\left(\phi_{t 1}, \phi_{t 2}\right.$, $\left.\phi_{t 3}\right)=\left(40^{\circ},-60^{\circ},-10^{\circ}\right),\left(\theta_{r 1}, \theta_{r 2}, \theta_{r 3}\right)=\left(50^{\circ}, 80^{\circ}, 20^{\circ}\right)$, $\left(\phi_{r 1}, \phi_{r 2}, \phi_{r 3}\right)=\left(10^{\circ}, 30^{\circ}, 60^{\circ}\right),\left(\gamma_{1}, \gamma_{2}, \gamma_{3}\right)=(\pi / 10, \pi / 4$, $9 \pi / 20),\left(\eta_{1}, \eta_{2}, \eta_{3}\right)=(\pi / 5,4 \pi / 5,2 \pi / 5)$ and $\left(f_{d 1}, f_{d 2}, f_{d 3}\right)=$ $(1,000,3,000,5,000) \mathrm{Hz}$.
First, we present the simulation results of 2D-DOD, 2DDOA, polarizations and Doppler frequency estimation for three targets when signal-to-noise ratio $(\mathrm{SNR})=10 \mathrm{~dB}$. The average of 100 Monte Carlo trials is shown in Table 1. From Table 1, it is observed that the estimates of seven parameters are close to their true values. The effectiveness and accuracy of the proposed algorithm can be verified from the simulation.

Further, the performance of the proposed algorithm is evaluated through root-mean-square error (RMSE). For estimating a parameter $\alpha$, the RMSE of the $p$ th target can be calculated by

$$
\operatorname{RMSE}(p)=\sqrt{\frac{1}{N_{t}} \sum_{t=1}^{N_{t}}\left(\hat{\alpha}_{p}^{(t)}-\alpha_{p}\right)^{2}},
$$

where $N_{t}$ is the number of Monte Carlo trials. Figure 2a,b,c,d,e,f,g respectively show the RMSE versus SNR for joint estimation of transmit elevation, transmit azimuth, receive elevation, receive azimuth, two polarization parameters and Doppler frequency. From Figure 2, we observe that the performance of multidimensional parameter estimation has been improved gradually with the increase of SNR. The seven parameters for each target can be efficiently obtained without peak searching, and the parameters for multiple targets can be paired automatically without wrong pairing. The additional pairing computation is eliminated using our algorithm. Based on the estimated multidimensional parameters, accurate identification and 3D localization of multiple targets can be achieved in MIMO radar.

Simulation 2. This simulation examines the estimates of 2D-DOD and 2D-DOA when there are $P=2$ closely spaced targets. SNR $=10 \mathrm{~dB}$. The two targets are diversely polarized with polarization parameters $\left(\gamma_{1}, \gamma_{2}\right)=(\pi / 10,9 \pi / 20), \quad\left(\eta_{1}, \eta_{2}\right)=(\pi / 5,4 \pi / 5)$. The

Table 1 Multidimensional parameters estimation result for three targets (SNR $=10 \mathrm{~dB})$

\begin{tabular}{|c|c|c|c|c|c|c|}
\hline \multirow{2}{*}{ Parameters } & \multicolumn{2}{|c|}{ Target 1} & \multicolumn{2}{|c|}{ Target 2} & \multicolumn{2}{|c|}{ Target 3} \\
\hline & True & Estimate & True & Estimate & True & Estimate \\
\hline$\theta_{t}$ & $10^{\circ}$ & $10.0069^{\circ}$ & $30^{\circ}$ & $29.9987^{\circ}$ & $70^{\circ}$ & $70.0012^{\circ}$ \\
\hline$\phi_{t}$ & $40^{\circ}$ & $39.9739^{\circ}$ & $-60^{\circ}$ & $-59.9708^{\circ}$ & $-10^{\circ}$ & $-10.0055^{\circ}$ \\
\hline$\theta_{r}$ & $50^{\circ}$ & $50.0054^{\circ}$ & $80^{\circ}$ & $80.0630^{\circ}$ & $20^{\circ}$ & $20.0075^{\circ}$ \\
\hline$\phi_{r}$ & $10^{\circ}$ & $9.9985^{\circ}$ & $30^{\circ}$ & $29.9829^{\circ}$ & $60^{\circ}$ & $60.0041^{\circ}$ \\
\hline$\gamma$ & $0.1 \pi$ & $0.0998 \pi$ & $0.25 \pi$ & $0.2493 \pi$ & $0.45 \pi$ & $0.4499 \pi$ \\
\hline$\eta$ & $0.2 \pi$ & $0.2003 \pi$ & $0.8 \pi$ & $0.7996 \pi$ & $0.4 \pi$ & $0.3996 \pi$ \\
\hline$f_{d}$ & $1,000 \mathrm{~Hz}$ & $1,000.3 \mathrm{~Hz}$ & $3,000 \mathrm{~Hz}$ & $2,999.8 \mathrm{~Hz}$ & $5,000 \mathrm{~Hz}$ & $4,999.9 \mathrm{~Hz}$ \\
\hline
\end{tabular}




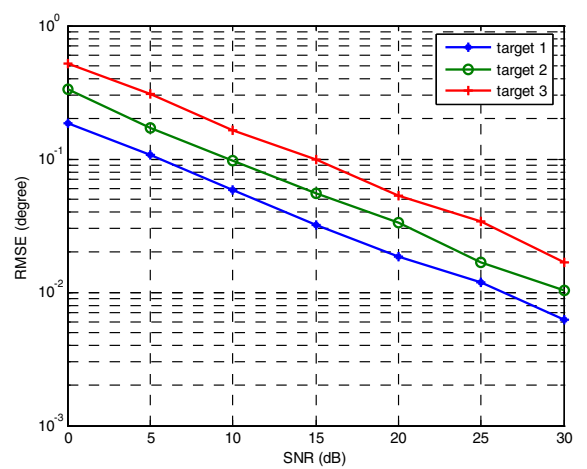

(a)

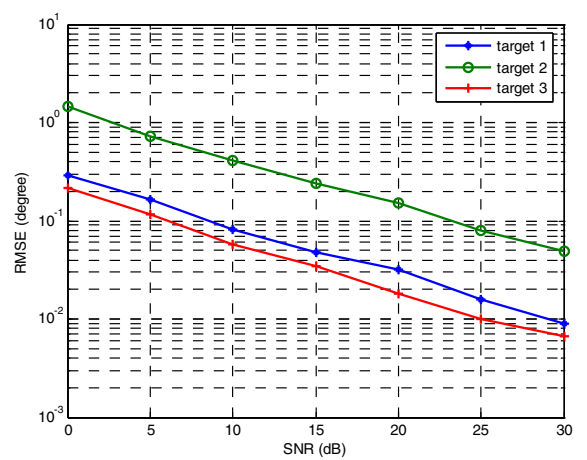

(c)

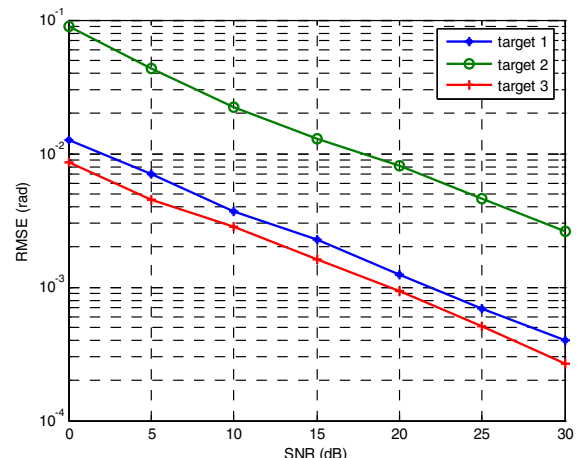

(e)

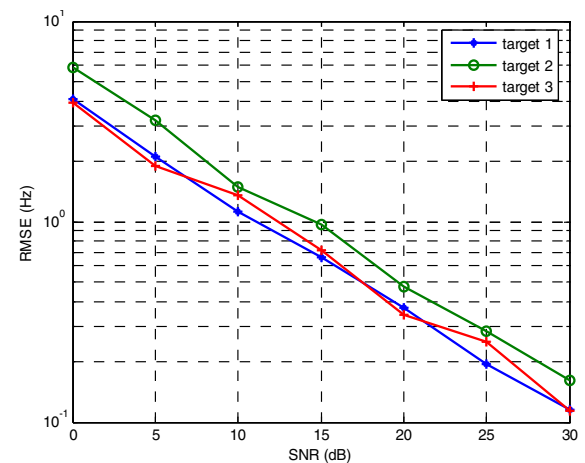

(g)

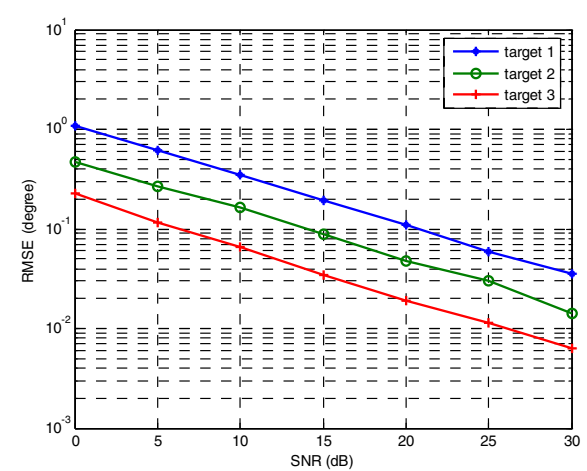

(b)

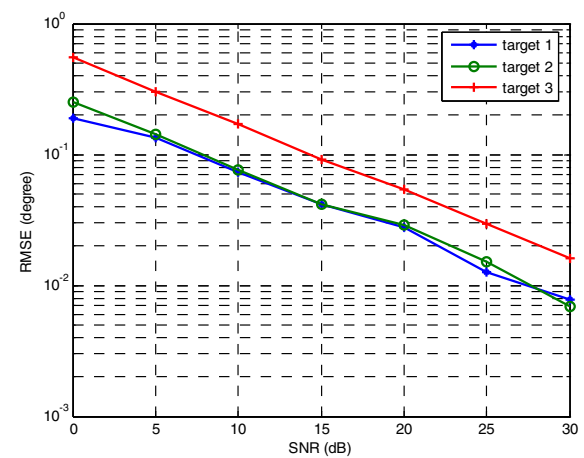

(d)

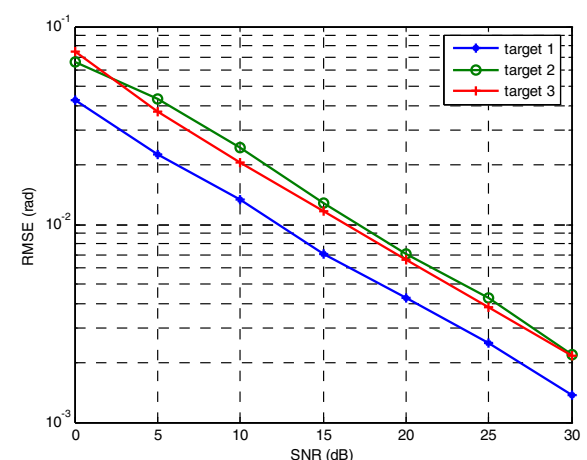

(f)

Figure 2 RMSE versus SNR for 2D-DOD, 2D-DOA, polarization parameters and Doppler frequency estimation. (a) transmit elevation $\theta_{t}$ (b) transmit azimuth $\phi_{t}, \mathbf{( c )}$ receive elevation $\theta_{r}$ (d) receive azimuth $\phi_{r},(\mathbf{e})$ polarization parameter $\gamma,(\mathbf{f})$ polarization parameter $\eta$ and $(\mathbf{g})$ Doppler frequency $f_{d}$. 
Doppler frequency is $\left(f_{d 1}, f_{d 2}\right)=(1,000,3,000) \mathrm{Hz}$. The following three scenarios are respectively considered.

Firstly, we assume that the two targets have close 2DDODs, i.e. $\left(\theta_{t 1}, \theta_{t 2}\right)=\left(10^{\circ}, 11^{\circ}\right),\left(\phi_{t 1}, \phi_{t 2}\right)=\left(40^{\circ}, 41^{\circ}\right)$ and close 2D-DOAs, i.e. $\left(\theta_{r 1}, \theta_{r 2}\right)=\left(50^{\circ}, 52^{\circ}\right),\left(\phi_{r 1}\right.$, $\left.\phi_{r 2}\right)=\left(68^{\circ}, 70^{\circ}\right)$. The average result of 100 Monte Carlo trials for 2D-DOD and 2D-DOA estimation is shown in Table 2.

Secondly, we assume that the two targets have the same 2D-DOD, i.e. $\left(\theta_{t 1}, \theta_{t 2}\right)=\left(10^{\circ}, 10^{\circ}\right),\left(\phi_{t 1}, \phi_{t 2}\right)=\left(40^{\circ}, 40^{\circ}\right)$ and close 2D-DOAs, i.e. $\left(\theta_{r 1}, \theta_{r 2}\right)=\left(30^{\circ}, 28^{\circ}\right),\left(\phi_{r 1}\right.$, $\left.\phi_{r 2}\right)=\left(57^{\circ}, 60^{\circ}\right)$. The average result of 100 Monte Carlo trials for 2D-DOD and 2D-DOA estimation is shown in Table 3.

Thirdly, we assume that the two targets have close 2D-DODs, i.e. $\left(\theta_{t 1}, \theta_{t 2}\right)=\left(10^{\circ}, 12^{\circ}\right),\left(\phi_{t 1}, \phi_{t 2}\right)=\left(30^{\circ}\right.$, $\left.32^{\circ}\right)$ and the same 2D-DOA, i.e. $\left(\theta_{r 1}, \theta_{r 2}\right)=\left(20^{\circ}, 20^{\circ}\right)$, $\left(\phi_{r 1}, \phi_{r 2}\right)=\left(80^{\circ}, 80^{\circ}\right)$. The average result of 100 Monte Carlo trials for 2D-DOD and 2D-DOA estimation is shown in Table 4.

The simulations of the above scenarios indicate that the proposed algorithm can distinguish multiple targets having close 2D-DODs and 2D-DOAs, or even the same 2D-DOD or 2D-DOA by polarization diversity. Therefore, it can uniquely identify the $2 \mathrm{D}$ transmit/receive angles of multiple closely spaced targets with high-resolution. However, traditional methods only contain angle parameter estimation without polarization information, leading to their performance degradation in locating multiple closely spaced targets.

Simulation 3. In this example, the proposed QALSbased algorithm is compared with TALS-based algorithm and ESPRIT-based algorithm to evaluate the performance of 2D-DOD and 2D-DOA estimation. For the other two algorithms, we extend the conventional PARAFAC TALSbased algorithm in [19] and the ESPRIT algorithm in [5] from 1D-estimation version to 2D-estimation version in order to compare them. Similarly, a uniform rectangular transmit array and a cross-dipoled uniform rectangular receive array are used in the two algorithms to estimate multidimensional parameters based on the signal model of polarimetric MIMO radar given in (12). In the extended

Table 2 2D angle estimation result when two targets have close 2D-DODs and close 2D-DOAs (SNR = $10 \mathrm{~dB}$ )

\begin{tabular}{cccccc}
\hline \multirow{2}{*}{ Parameters } & \multicolumn{2}{c}{ Target 1 } & & \multicolumn{2}{c}{ Target 2 } \\
\cline { 2 - 3 } \cline { 6 - 6 } & True & Estimate & & True & Estimate \\
\hline$\theta_{t}$ & $10^{\circ}$ & $10.0040^{\circ}$ & & $11^{\circ}$ & $10.9966^{\circ}$ \\
$\phi_{t}$ & $40^{\circ}$ & $40.0082^{\circ}$ & & $41^{\circ}$ & $40.9680^{\circ}$ \\
$\theta_{r}$ & $50^{\circ}$ & $50.0184^{\circ}$ & & $52^{\circ}$ & $52.0120^{\circ}$ \\
$\phi_{r}$ & $68^{\circ}$ & $67.9970^{\circ}$ & & $70^{\circ}$ & $69.9983^{\circ}$ \\
\hline
\end{tabular}

Table $32 \mathrm{D}$ angle estimation result when two targets have the same 2D-DOD and close 2D-DOAs (SNR = $10 \mathrm{~dB}$ )

\begin{tabular}{cccccc}
\hline \multirow{2}{*}{ Parameters } & \multicolumn{2}{c}{ Target 1 } & & \multicolumn{2}{c}{ Target 2 } \\
\cline { 2 - 3 } \cline { 6 - 7 } & True & Estimate & & True & Estimate \\
\hline$\theta_{t}$ & $10^{\circ}$ & $9.9723^{\circ}$ & & $10^{\circ}$ & $10.0389^{\circ}$ \\
$\phi_{t}$ & $40^{\circ}$ & $39.7819^{\circ}$ & & $40^{\circ}$ & $40.1559^{\circ}$ \\
$\theta_{r}$ & $30^{\circ}$ & $29.9974^{\circ}$ & & $28^{\circ}$ & $28.0003^{\circ}$ \\
$\phi_{r}$ & $57^{\circ}$ & $56.9936^{\circ}$ & & $60^{\circ}$ & $60.0105^{\circ}$ \\
\hline
\end{tabular}

TALS-based algorithm, the observed matrix $\mathbf{Y}$ in (12) is written as

$$
\mathbf{Y}=\mathbf{A}_{t}\left(\theta_{t}, \phi_{t}\right) \diamond \tilde{\mathbf{A}}_{r}\left(\theta_{r}, \phi_{r}, \gamma, \eta\right) \mathbf{B}^{T}\left(f_{d}\right)+\mathbf{N}
$$

where $\tilde{\mathbf{A}}_{r}\left(\theta_{r}, \phi_{r}, \gamma, \eta\right)=\mathbf{A}_{r}\left(\theta_{r}, \phi_{r}\right) \diamond \mathbf{G}\left(\theta_{r}, \phi_{r}, \gamma, \eta\right)$. From (44), it is shown that $\mathbf{Y}$ is a PARAFAC trilinear decomposition model. Thus, TALS-based algorithm in [19] can be applied to recover the three parameter matrices $\mathbf{A}_{t}\left(\theta_{t}, \phi_{t}\right)$, $\tilde{\mathbf{A}}_{r}\left(\theta_{r}, \phi_{r}, \gamma, \eta\right)$ and $\mathbf{B}\left(f_{d}\right)$. Following this, 2D-DOD, 2DDOA as well as polarization and Doppler frequency can be estimated exploiting the Vandermonde structure and Kronecker matrix products in the three parameter matrices. The extended ESPRIT-based algorithm employs the shift invariance property of transmit and receive arrays, in which both the steering vectors of transmit and receive arrays have the form of kronecker product for the rectangular arrays, as shown in (6). The signal subspace can be obtained by eigen-value decomposition of covariance matrix of the observed matrix $\mathbf{Y}$ in (12). Then, multidimensional parameters can be estimated by properly partitioning the signal subspace matrix into different submatrices [16]. The procedure of pairing 2D-DODs and 2D-DOAs for multiple targets is performed similarly to [30].

In simulation 3, we consider a scenario that there are $P=2$ targets. Their polarization parameters and Doppler frequency are $\left(\gamma_{1}, \gamma_{2}\right)=(\pi / 10, \pi / 4),\left(\eta_{1}, \eta_{2}\right)=$ $(\pi / 5,4 \pi / 5)$ and $\left(f_{d 1}, f_{d 2}\right)=(1,000,3,000) \mathrm{Hz}$. We firstly evaluate these three algorithms in the condition of targets with different 2D-DODs and 2D-DOAs. Assume that they

Table 4 2D angle estimation result when two targets have the same 2D-DOA and close 2D-DODs (SNR = $10 \mathrm{~dB}$ )

\begin{tabular}{ccccccc}
\hline \multirow{2}{*}{ Parameters } & \multicolumn{2}{c}{ Target 1 } & & \multicolumn{2}{c}{ Target 2 } \\
\cline { 2 - 3 } \cline { 6 - 7 } & True & Estimate & & True & Estimate \\
\hline$\theta_{t}$ & $10^{\circ}$ & $10.0125^{\circ}$ & & $12^{\circ}$ & $11.9841^{\circ}$ \\
$\phi_{t}$ & $30^{\circ}$ & $29.9970^{\circ}$ & & $32^{\circ}$ & $31.9930^{\circ}$ \\
$\theta_{r}$ & $20^{\circ}$ & $19.9717^{\circ}$ & & $20^{\circ}$ & $20.0278^{\circ}$ \\
$\phi_{r}$ & $80^{\circ}$ & $79.9013^{\circ}$ & & $80^{\circ}$ & $80.1042^{\circ}$ \\
\hline
\end{tabular}


are located at $\left(\theta_{t 1}, \theta_{t 2}\right)=\left(10^{\circ}, 20^{\circ}\right),\left(\phi_{t 1}, \phi_{t 2}\right)=\left(50^{\circ}\right.$, $\left.60^{\circ}\right), \quad\left(\theta_{r 1}, \theta_{r 2}\right)=\left(30^{\circ}, 40^{\circ}\right), \quad\left(\phi_{r 1}, \phi_{r 2}\right)=\left(70^{\circ}, 80^{\circ}\right)$. The performance comparison of transmit elevation/azimuth (2D-DOD) and receive elevation/azimuth (2D-DOA) among the three algorithms is respectively shown in Figure $3 a, b, c, d$, where the RMSE is the average of the two targets. Figure 3 reveals that the three algorithms have close estimation performance in this condition, with QALS-based algorithm being a little better than the other two algorithms. Next, we evaluate the three algorithms in the condition of targets with the same 2D-DOA. Assume that they are located at $\left(\theta_{t 1}, \theta_{t 2}\right)=$ $\left(10^{\circ}, 20^{\circ}\right), \quad\left(\phi_{t 1}, \phi_{t 2}\right)=\left(50^{\circ}, 60^{\circ}\right), \quad\left(\theta_{r 1}, \theta_{r 2}\right)=$ $\left(40^{\circ}, 40^{\circ}\right), \quad\left(\phi_{r 1}, \phi_{r 2}\right)=\left(80^{\circ}, 80^{\circ}\right)$. The performance comparison is respectively shown in Figure 4a,b,c,d. Figure 4 demonstrates that all the three algorithms can identify two targets with the same 2D-DOA since the receive array manifolds for the two targets are distinguishable by polarization diversity. However, the performance of QALS-based algorithm outperforms that of the other two

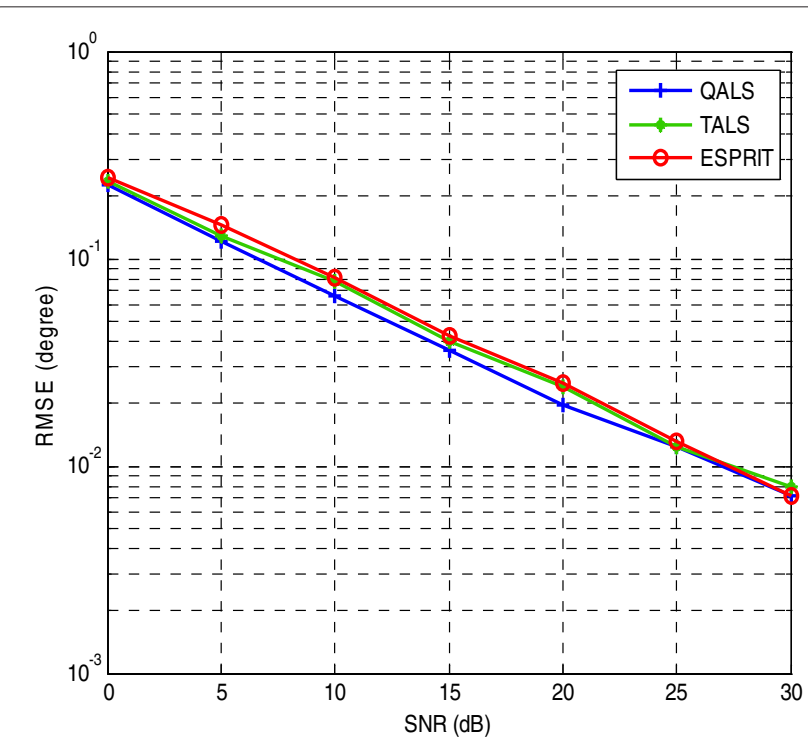

(a)

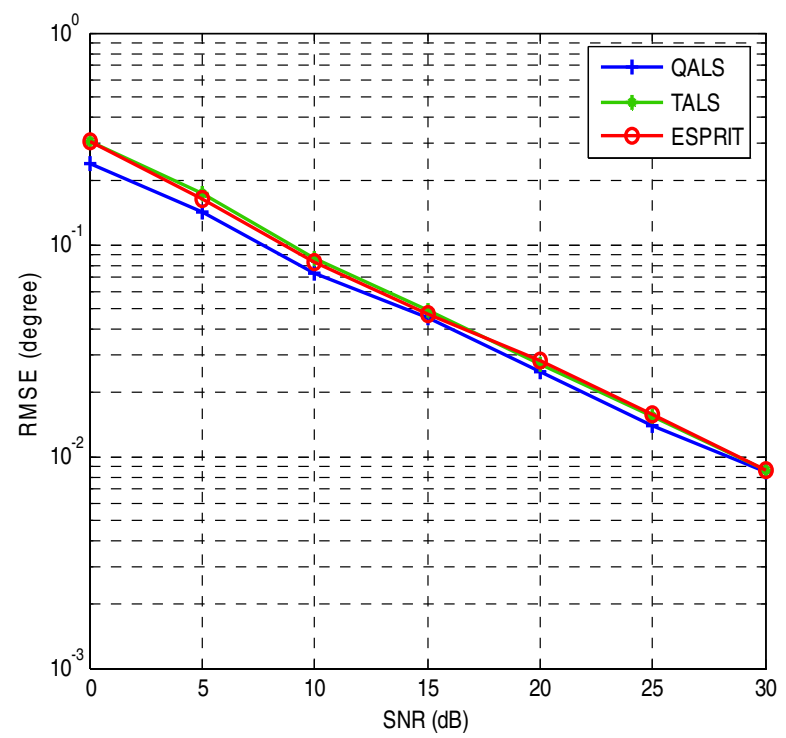

(c)

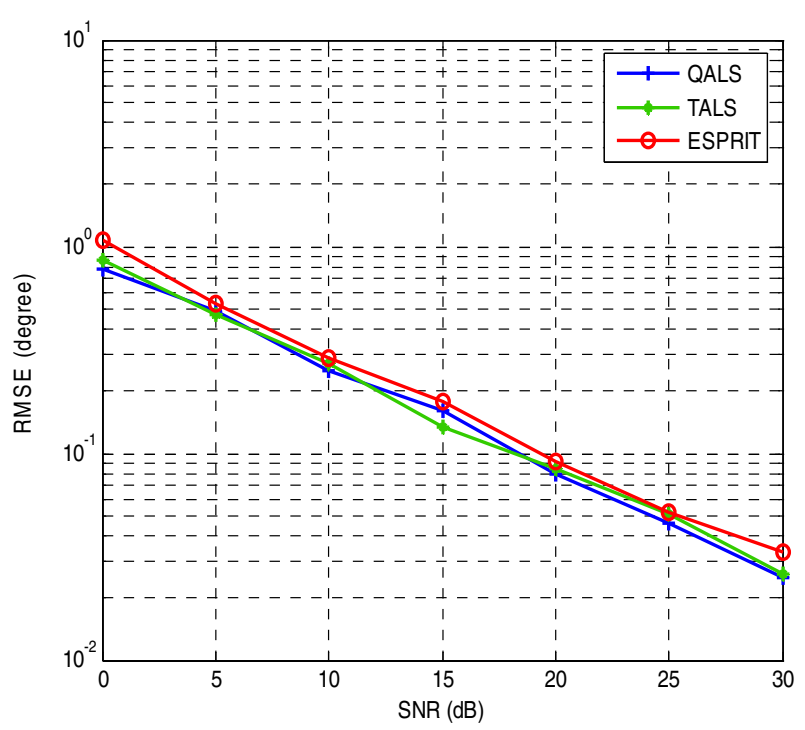

(b)

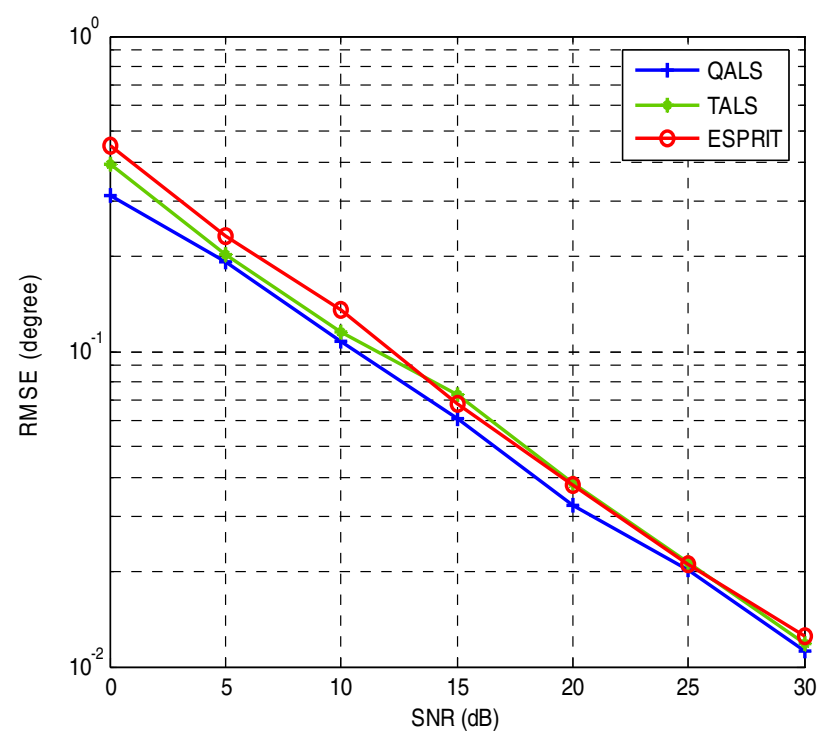

(d)

Figure 3 Performance comparison of QALS-based algorithm with TALS-based algorithm and ESPRIT-based algorithm. Two targets have different 2D-DODs and 2D-DOAs. (a) transmit elevation $\theta_{t}$ (b) transmit azimuth $\phi_{t}$ (c) receive elevation $\theta_{r}$ and (d) receive azimuth $\phi_{r}$. 
algorithms, especially for 2D-DOA estimation. We finally evaluate the three algorithms in the condition of targets with the same 2D-DOD. Assume that they are located at $\left(\theta_{t 1}, \theta_{t 2}\right)=\left(10^{\circ}, 10^{\circ}\right), \quad\left(\phi_{t 1}, \phi_{t 2}\right)=\left(50^{\circ}, 50^{\circ}\right), \quad\left(\theta_{r 1}, \theta_{r 2}\right)=$ $\left(30^{\circ}, 40^{\circ}\right), \quad\left(\phi_{r 1}, \phi_{r 2}\right)=\left(70^{\circ}, 80^{\circ}\right)$. The performance comparison is respectively shown in Figure $5 \mathrm{a}, \mathrm{b}, \mathrm{c}, \mathrm{d}$. From Figure 5, we can see that in this condition, TALS-based algorithm and ESPRIT-based algorithm cannot work in receive elevation/azimuth estimation because of the rank deficiency. Also, for transmit elevation/azimuth estimation, the RMSE of QALS-based algorithm is smaller than that of the other two algorithms. The reason is that for the QALS-based method, the Kruskal-rank condition of qudrilinear decomposition in (42) is well satisfied in the scenario that $P$ targets have the same 2D-DOD. Thus, multidimensional parameters can be uniquely identified, and the proposed algorithm still works well in this scenario, whereas the TALS-based algorithm and ESPRITbased algorithm have severe performance deterioration. In addition, for the ESPRIT-based algorithm, an additional multidimensional parameter pairing for multiple targets is needed.

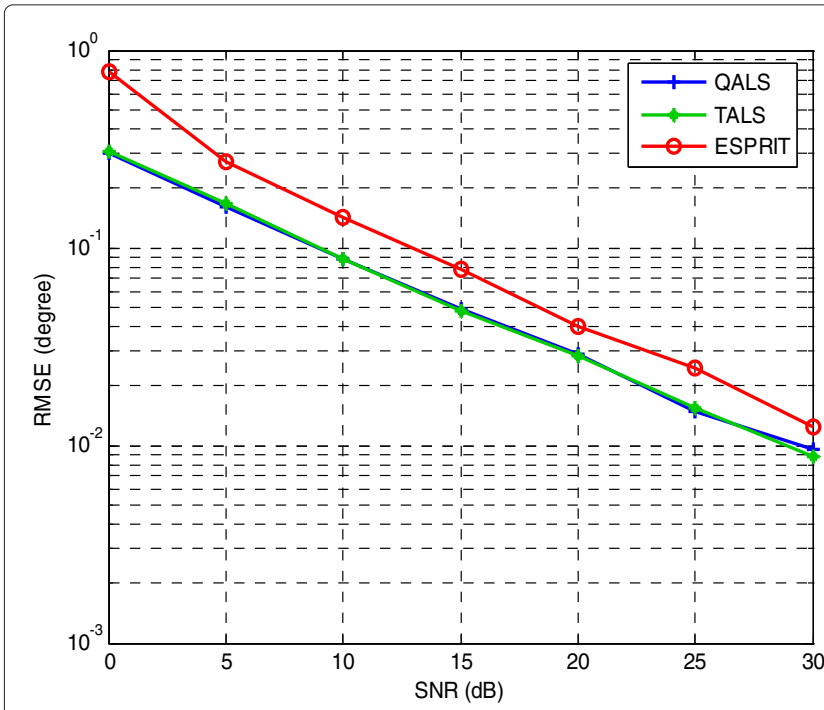

(a)

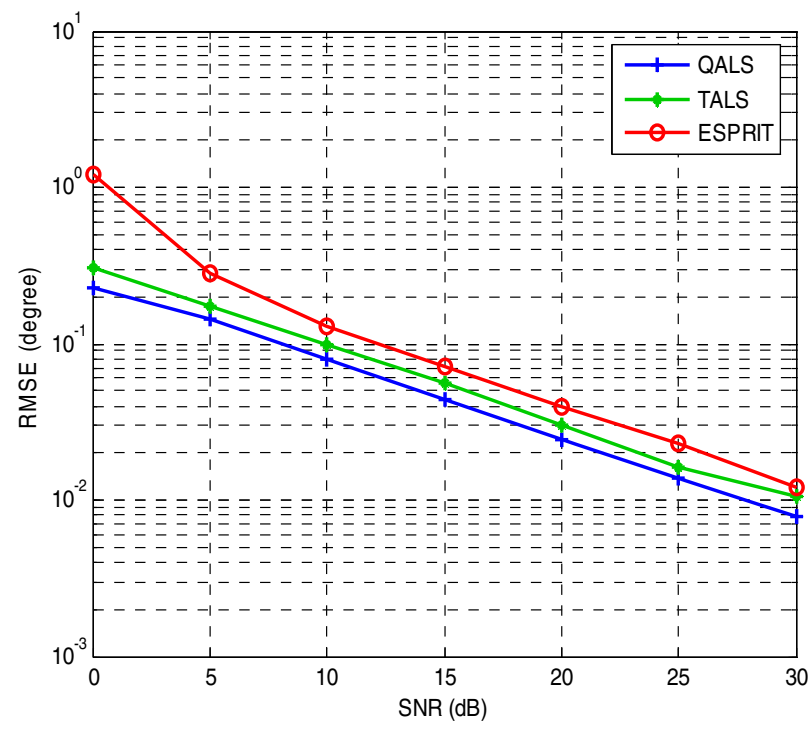

(c)

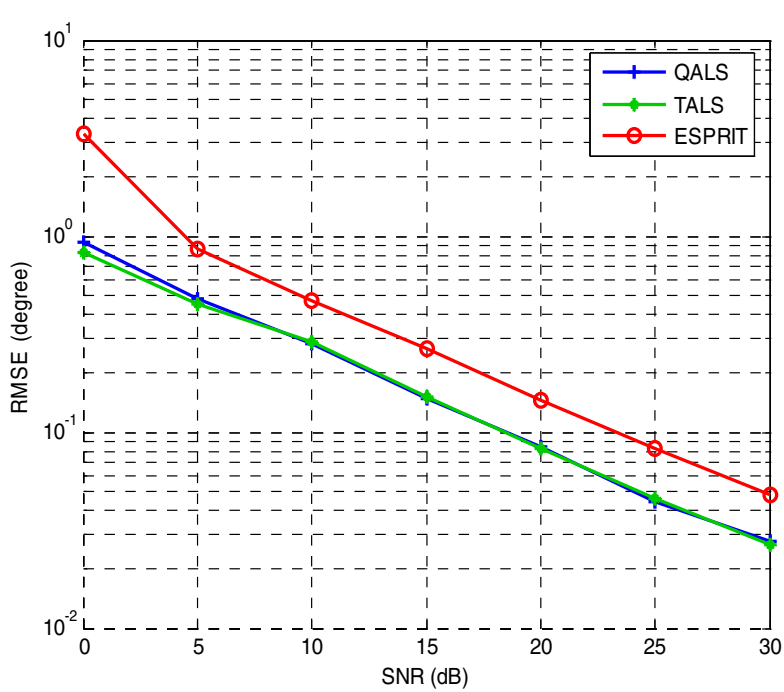

(b)

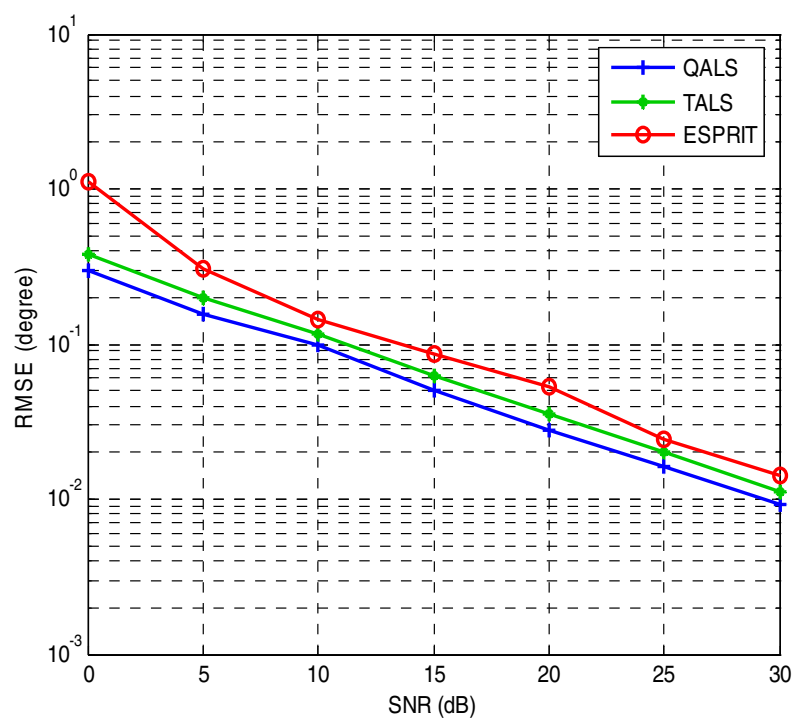

(d)

Figure 4 Performance comparison of QALS-based algorithm with TALS-based algorithm and ESPRIT-based algorithm. Two targets have the same 2D-DOA. (a) transmit elevation $\theta_{t}$ (b) transmit azimuth $\phi_{t,}$ (c) receive elevation $\theta_{r}$ and (d) receive azimuth $\phi_{r}$. 


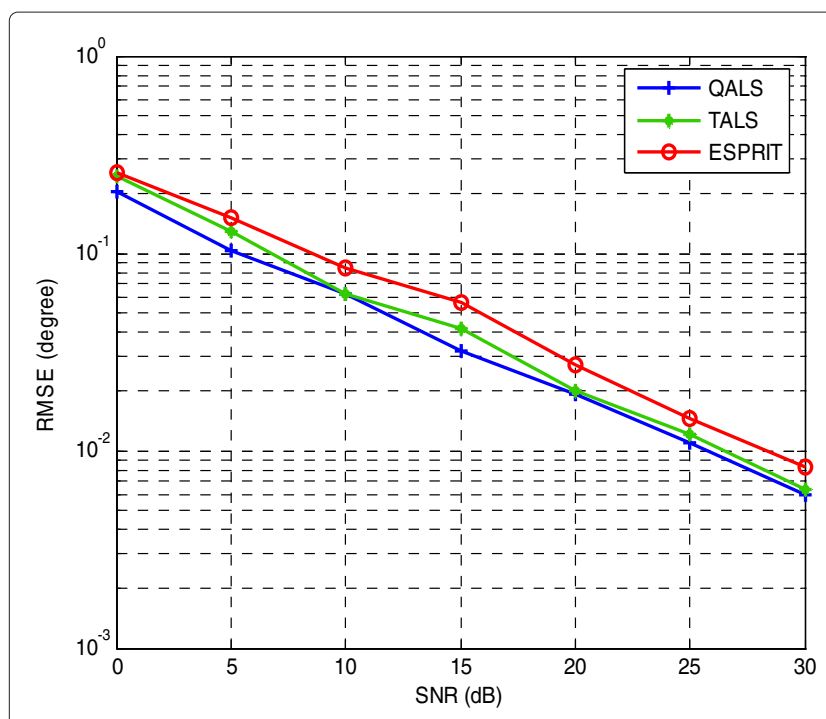

(a)

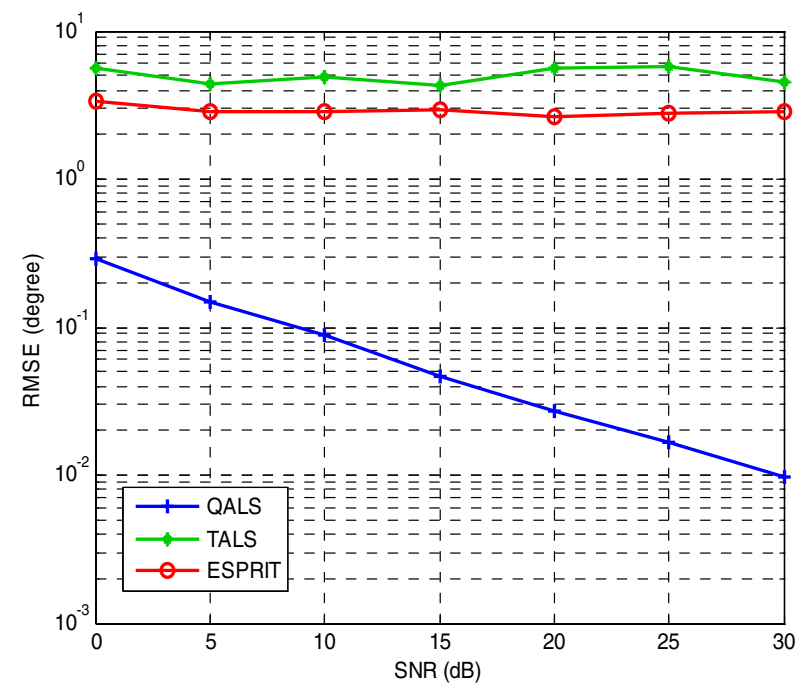

(c)

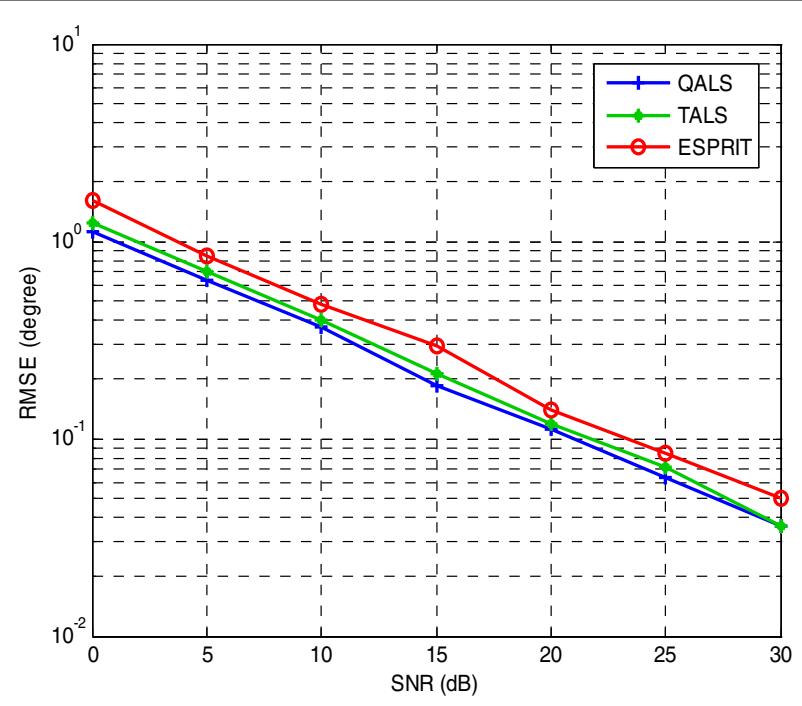

(b)

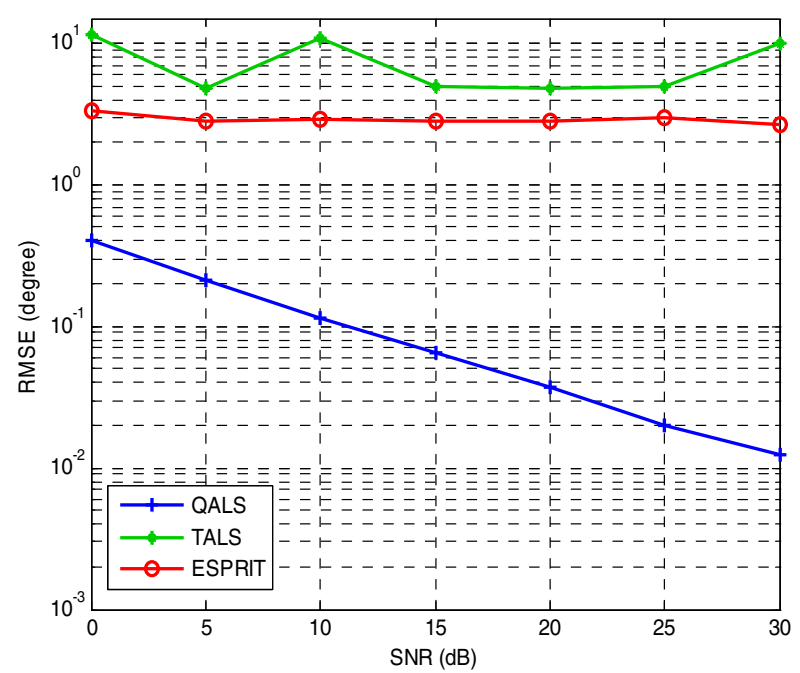

(d)

Figure 5 Performance comparison of QALS-based algorithm with TALS-based algorithm and ESPRIT-based algorithm. Two targets have the same 2D-DOD. (a) transmit elevation $\theta_{t}$ (b) transmit azimuth $\phi_{t,}$ (c) receive elevation $\theta_{r}$ and (d) receive azimuth $\phi_{r}$

\section{Conclusions}

In this article, we investigate multidimensional target parameter estimation in a polarimetric bistatic MIMO radar system using PARAFAC quadrilinear decomposition. The signal model has been developed and a novel algorithm for joint estimation of 2D-DOA, 2D-DOD, polarization parameters and Doppler frequency has been presented based on QALS. The algorithm has many merits: (i) it requires neither multidimensional spectral peak searching nor covariance matrix estimation and several eigen-decompositions that may bring error accumulation, which enhances the accuracy of estimation; (ii) multidimensional parameters can be well paired automatically, which reduces the complexity of additional pairing; (iii) it can distinguish multiple targets having close 2D-DODs and 2D-DOAs or even the same 2D-DOD or 2D-DOA by exploiting polarization diversity and the uniqueness of quadrilinear decomposition. Thus, the performance of multi-target 2D-DOD and 2D-DOA estimation in polarimetric MIMO radar has been greatly improved; (iv) unlike the previous algorithms with only respect to 1D-DOD and 1D-DOA estimation in bistatic MIMO radar, the proposed algorithm can obtain 2D-DOA and 2D-DOD estimation with high-resolution, which is of importance for accurate identification and 3D localization of multiple targets in MIMO radar. 


\section{Competing interests}

The authors declare that they have no competing interests.

\section{Acknowledgements}

The work is supported by the National Natural Science Foundation of China (No. 61071140). The authors wish to thank the anonymous reviewers for their valuable comments and suggestions which greatly improved the manuscript.

Received: 6 July 2012 Accepted: 19 July 2013

Published: 2 August 2013

\section{References}

1. E Fisher, A Haimovich, R Blum, D Chizhik, L Cimini, R Valenzuela. MIMO radar: an idea whose time has come, in Proc. IEEE Radar Conf., Philadelphia, 26-29 April (IEEE Piscataway, 2004), pp. 71-78

2. I Bekkerman, J Tabrikian, Target detection and localization using MIMO radars and sonars. IEEE Trans. Signal Process. 54(10), 3873-3883 (2006)

3. J Li, P Stoica, MIMO Radar Signal Processing. (Wiley, New York, 2009)

4. H Yan, J Li, G Liao, Multitarget identification and localization using bistatic MIMO radar systems. EURASIP J. Adv. Signal Process. 2008, 283483 (2008)

5. C Duofang, C Baixiao, Q Guodong, Angle estimation using ESPRIT in MIMO radar. Electron. Lett. 44(12), 770-771 (2008)

6. M Jin, G Liao, J Li, Joint DoD and DoA estimation for bistatic MIMO radar. Signal Process. 89(2), 244-251 (2009)

7. J Chen, H Gu, W Su, A new method for joint DOD and DOA estimation in bistatic MIMO radar. Signal Process. 90, 714-719 (2010)

8. ML Bencheikh, Y Wang, Polynomial root finding technique for joint DOA DOD estimation in bistatic MIMO radar. Signal Process. 90(9), 2723-2730 (2010)

9. ML Bencheikh, Y Wang, Joint DOD-DOA estimation using combined ESPRIT-MUSIC approach in MIMO radar. Electron. Lett. 46(15), 1081-1083 (2010)

10. X Zhang, $L X u, L X u, D X u$, Direction of Departure (DOD) and Direction of Arrival (DOA) estimation in MIMO radar with reduced-dimension MUSIC. IEEE Commun. Lett. 14(12), 1161-1163 (2010)

11. YD Zhang, MG Amin, B Himed, Joint DOD/DOA estimation in MIMO radar exploiting time-frequency signal representations. EURASIP J. Adv. Signal Process. 2012(102) (2012)

12. CYunhe, Joint estimation of angle and Doppler frequency for bistatic MIMO radar. Electron. Lett. 46(2), 170-172 (2010)

13. J Gong, H Lv, Y Guo. Multidimensional parameters estimation for bistatic MIMO radar, in Proc. 7th International Conf., Wireless Communications, Networking and Mobile Computing (WiCoM, 2011) Wuhan, 23-25, Sept 2011 (Piscataway, 2011), pp. 1-4

14. J Zhang, Z Zheng, X Li, An algorithm for DOD-DOA and Doppler frequency jointly Estimating of bistatic MIMO radar. J. Electron Inf. Technol, (In Chinese). 32(8), 1843-1848 (2010)

15. H Jiang, DF Wang, C Liu, Joint parameter estimation of DOD/DOA/polarization for bistatic MIMO radar. J. China Universities Posts Telecommunications. 17(5), 32-37 (2010)

16. H Jiang, DF Wang, C Liu. Estimation of DOD and 2D-DOA and polarizations for bistatic MIMO radar, in Proc. 19th Annual Wireless and Optical Communications Conf., Shanghai, 14-15 May, 2010 (IEEE Piscataway, 2010), pp. 1-5

17. ND Sidiropoulos, GB Giannakis, R Bro, Blind PARAFAC receivers for DS-CDMA systems. IEEE Trans. Signal Process. 48(3), 810-823 (2000)

18. ND Sidropoulos, R Bro, GB Giannakos, Parallel factor analysis in sensor array processing. IEEE Trans. Signal Process. 48(8), 2377-2388 (2000)

19. D Nion, ND Sidiropoulos, Tensor algebra and multidimensional harmonic retrieval in signal processing for MIMO Radar. IEEE Trans Signal Process. 58(11), 5693-5705 (2010)

20. X Zhang, Z Xu, L Xu, D Xu, Trilinear decomposition-based transmit angle and receive angle estimation for multiple-input multiple-output radar. IET Radar Sonar Navigation. 5(6), 626-631 (2011)

21. D Nion, ND Sidiropoulos, Adaptive algorithms to track the PARAFAC decomposition of a third-order tensor. IEEE Trans. Signal Process. 57(6), 2299-2310 (2009)

22. X Liu, ND Sidiropoulos, Cramer Rao lower bounds for low-rank decomposition of multidimensional arrays. IEEE Trans. Signal Process. 49(9), 2074-2086 (2011)
23. Y Shi, X Zhang, Quadrilinear decomposition-based blind signal detection for polarization sensitive uniform square array. Prog. Electromagnetics Res. 83, 263-278 (2008)

24. J Li, RT Compton, Two-dimensional angle and polarization estimation using the ESPRIT algorithm. IEEE Trans. Antennas Propagation. 40(5), 550-555 (1992)

25. ND Sidropoulos, R Bro, On the uniqueness of multilinear decomposition of N-way arrays. J. Chemometrics. 14, 229-239 (2000)

26. GH Golub, CFV Loan, Matrix Computations, 3rd edn. (The Johns Hopkins University, Baltimore, 1996)

27. N Fourikis, Advanced Array Systems, Applications and RF Technologies. (Academic, London NW, 2000)

28. SA Vorobyov, Y Rong, ND Sidiropoulos, AB Gershman, Robust iterative fitting of multilinear models. IEEE Trans. Signal Process. 53(8), 2678-2689 (2005)

29. MI Skolnik, Introduction to Radar System, 3rd edn. (Academic, London NW, 2001)

30. J Chen, H Gu, W Su, Angle estimation using ESPRIT without pairing in MIMO radar. Electron. Lett. 44(24), 1422-1423 (2008)

doi:10.1186/1687-6180-2013-133

Cite this article as: Jiang et al:: A PARAFAC-based algorithm for multidimensional parameter estimation in polarimetric bistatic MIMO radar. EURASIP Journal on Advances in Signal Processing 2013 2013:133.

\section{Submit your manuscript to a SpringerOpen ${ }^{\circ}$ journal and benefit from:}

- Convenient online submission

- Rigorous peer review

- Immediate publication on acceptance

- Open access: articles freely available online

- High visibility within the field

- Retaining the copyright to your article

Submit your next manuscript at $>$ springeropen.com 